\title{
Cohérence électroencéphalographique, vivacité des souvenirs et effets de valence émotionnelle des mouvements oculaires bilatéraux pendant le rappel de souvenirs désagréables et l'association libre subséquente : implications pour l'EMDR
}

\author{
Matthew Yaggie \\ Larry Stevens \\ Seth Miller \\ Angela Abbott \\ Chad Woodruff \\ Mike Getchis \\ Sean Stevens \\ Leslie Sherlin \\ Brandon Keller \\ Suzanne Daiss \\ Faculté des sciences psychologiques ; Université de l'Arizona du Nord
}

\begin{abstract}
Cette étude a examiné les effets de la stimulation bilatérale pendant le rappel de souvenirs désagréables suivi d'association libre, de manière similaire à la phase 4 de la psychothérapie EMDR. Quarante-six sujets sains féminins ont été affectés de manière aléatoire à l'une des trois conditions : mouvements oculaires bilatéraux (MOB), yeux fixes avec des mouvements en arrière-plan (MAP), ou contrôle par yeux fixes (Point), chacune se déroulant avec le rappel d'un souvenir modérément désagréable et chacune étant suivie d'une association libre avec le souvenir. Les enregistrements électroencéphalographiques ont été effectués sur ces participantes pendant la minute d'association libre avec le souvenir initial après chacune des cinq administrations des conditions. Les résultats n'ont révélé que des tendances à la hausse de la cohérence interhémisphérique bêta après les MOB. Toutefois, des augmentations statistiquement significatives des cohérences thêta et bêta frontales droites ont été montrées après les $\mathrm{MOB}$, avec des tendances à la hausse similaires pour les thêta et bêta frontales gauches et pour les gamma frontales droites. Les cartes d'électrodes corticaux sont présentées pour ces effets de cohérence bêta. Les évaluations de vivacité de l'imagerie et de la valence émotionnelle ont été recueillies après chaque série de mouvements oculaires plus les associations libres, et ont montré une diminution significative pour toutes les conditions. Les résultats sont discutés dans le contexte de la proposition d'un modèle intégré de cohérence corticale en 2 étapes. Des suggestions sont formulées pour les recherches futures, y compris une investigation des implications possibles pour le traitement des traumatismes crâniens.
\end{abstract}

Mots-clés : EMDR (désensibilisation et retraitement par les mouvements oculaires) ; électroencéphalographie (EEG) ; cohérence ; stimulation bilatérale ; état de stress post-traumatique (ESPT) ; traumatisme crânien (TC)

This article originally appeared as Yaggie, M., Stevens, L., Miller, S., Abbott, A., Woodruff, C., Getchis, M., Stevens, S., Sherlin, L., Keller, B., \& Daiss, S. (2015). Electroencephalography Coherence, Memory Vividness, and Emotional Valence Effects of Bilateral Eye Movements During Unpleasant Memory Recall and Subsequent Free Association: Implications for Eye Movement Desensitization and Reprocessing. Journal of EMDR Practice and Research, 9(2), 78-97. Translated by Jenny Ann Rydberg. 
U ne quantité d'investigations de recherche soigneusement conçues se sont accumulées au cours des 15 dernières années, prouvant les effets des seuls mouvements oculaires bilatéraux (MOB) sur plusieurs processus cognitifs. Des études couvrant une large palette de tests de mémoire (voir Propper \& Christman, 2008, pour une analyse complète de cette littérature) ont documenté de manière convaincante l'amélioration subséquente du rappel de souvenirs épisodiques après un temps de MOB. Et plus récemment encore, Samara, Elzinga, Slagter et Nieuwenhuis (2011) et Nieuwenhuis et coll. (2013) ont démontré à travers quatre études distinctes un rappel amélioré de mots à valence négative après une stimulation bilatérale.

Une littérature apparemment contradictoire existe également, documentant une vivacité et une valence émotionnelle positives et négatives réduites des souvenirs immédiatement après des MOB. Toutefois, dans ces études, les MOB sont effectués pendant que le participant garde en mémoire de travail l'expérience ciblée et le rappel est testé peu après. Cette " déflation imaginaire " est posée hypothétiquement comme se produisant en raison d'une saturation de la mémoire de travail avec à la fois l'expérience mnésique et la tâche MOB, produisant un floutage et un affaiblissement de la vivacité et de la valence émotionnelle subséquentes (Andrade, Kavanagh \& Baddeley, 1997 ; Gunter \& Bodner, 2008). Il faut toutefois noter que la vivacité des souvenirs dans ces études n'était pas examinée après une période de retraitement ou d'association libre comme c'est le cas dans la phase 4 de la psychothérapie EMDR.

Une seule étude a examiné la vivacité des souvenirs après la désensibilisation de la phase 4 dans la pratique EMDR. Lee et Drummond (2008) ont invité 48 participants sains à se rappeler une expérience modérément perturbante, ont activé ce souvenir pendant une condition de mouvements oculaires ou de regard fixe, puis ont entamé une période de retraitement avec distanciation ou avec reviviscences, en répétant ces étapes jusqu'à ce que le niveau de perturbation soit égal à zéro ou que 45 minutes se soient écoulées. La vivacité des souvenirs était évaluée au début et à la fin de la phase de désensibilisation et après une semaine. Les résultats ont révélé une diminution significative de la vivacité des souvenirs seulement pour les conditions de mouvements oculaires et de distanciation, et seulement immédiatement après la désensibilisation, ce qui est globalement cohérent avec le mécanisme de changement supposé de l'EMDR.

\section{Neuromécanismes proposés sous-tendant le composant des mouvements oculaires bilatéraux de l'EMDR}

Les mécanismes qui sous-tendent le(s) rôle(s) des MOB dans le traitement du trauma en EMDR demeurent inconnus. Cette incertitude se poursuit malgré plus de deux décennies de formulation d'hypothèses, de développement de modèles et de recherches dirigées. Après la suggestion de Shapiro (1989) selon laquelle les MOB activent un mécanisme de type mouvements oculaires rapides (MOR) pour relier des réseaux neuraux qui ont échoué à s'associer pendant la création du trauma, au moins douze hypothèses distinctes ont été avancées pour expliquer la contribution des MOB au traitement du trauma.

Voici une brève présentation chronologique de ces diverses hypothèses :

(a) Bien sûr, l'hypothèse du traitement accéléré (devenu adaptatif) de l'information de Shapiro (1989) était le premier modèle fonctionnel de l'EMDR. Comme mentionné dans le texte ciaprès, ce modèle proposait que des réseaux neuraux dissociés par le trauma deviennent de nouveau associés à des informations cognitives et émotionnelles plus constructives par le biais de la stimulation bilatérale (SBL) et des mécanismes de retraitement subséquents ;

(b) Dyck (1993) a ensuite suggéré que l'EMDR fonctionne par l'extinction des conduites de fuite/évitement et en reconditionnant à des stimuli neutres ;

(c) Denny (1995) a suggéré un modèle de l'inhibition dans lequelles souvenirs traumatiques peuventêtre inhibés par une réponse d'orientation aux MOB ;

(d) Armstrong et Vaughan (1996) ont expliqué, de manière similaire, que la réponse d'orientation sert à éteindre et à permettre une réévaluation des stimuli non conditionnés ;

(e) MacCulloch et Feldman (1996) ont proposé que les MOB établissent un "réflexe de réassurance " à partir d'éléments viscéraux positifs de la réponse d'orientation ;

(f) Andrade et coll. (1997) ont suggéré que les MOB altèrent la vivacité des expériences traumatiques sur le calepin visuospatial de la mémoire de travail ;

(g) Bergmann (1998) a suggéré que les MOB réinitialisent les cellules pacemaker du noud septal, conduisant à une connectivité interhémisphérique fonctionnelle améliorée ;

(h) Bergmann (2000) a ensuite proposé un modèle neuroanatomique dans lequel les MOB intègrent les souvenirs traumatiques dans des réseaux 
sémantiques et néocorticaux autres par des voies cérébelleuses et thalamocorticales ;

(i) Servan-Schreiber (2000) a proposé que les MOB induisent un arrière-plan d'activité neurologique interhémisphérique synchrone qui relie fonctionnellement des aires précédemment déconnectées ;

(j) Stickgold (2002) a expliqué le rôle des MOB dans l'intégration des souvenirs traumatiques dans des réseaux sémantiques à travers une réduction à médiation cholinergique des souvenirs épisodiques de l'hippocampe et des affects négatifs amygdaliens ;

(k) Corrigan (2002) a proposé que les MOB rétablissent les associations bilatérales entre les sous-divisions cognitives et affectives du cortex cingulaire antérieur ;

(1) Rasolkhani-Kalhorn et Harper (2006) ont suggéré que les saccades de basse fréquence des $\mathrm{MOB}$ dépotentialisent les synapses limbiques mnésiques de la peur.

Vu le nombre d'hypothèses différentes proposées pour expliquer l'efficacité du composant MOB de l'EMDR, il n'est pas étonnant que les résultats des recherches n'aient pas encore soutenu un modèle plutôt qu'un autre, ni intégré ces modèles en une explication complète du composant MOB. De plus, la complexité neuroanatomique et la nature abstraite de plusieurs des postulats de ces modèles rendent leur validation auprès de populations cliniques très difficiles.

\section{Effets de l'EMDR sur la dynamique cérébrale}

Les études de neuroimagerie ont identifié de manière convaincante un processus pathologique qui correspondrait aux mécanismes principaux de l'expérience de l'ESPT : une régulation réduite par les aires préfrontales médiales et le cortex cingulaire antérieur d'une amygdale excessivement active (Bremner, 2007 ; Francati, Vermetten \& Bremner, 2007). Par conséquent, on pourrait s'attendre à ce que les thérapies visant à améliorer les effets des expériences traumatiques aient un impact correcteur inverse sur ces mêmes aires. Bien que des études de neuroimagerie à groupes contrôlés sur la psychothérapie EMDR soient assez rares et diverses et qu'elles impliquent des nombres très réduits de participants (2-10) dans les groupes de traitement, les résultats ont été généralement cohérents. Dans trois études de groupes de tomographie d'émission monophotonique (TEMP, dite aussi SPECT), deux d'imagerie à résonance magnétique (IRM) et quatre d'électroencéphalographie (EEG) analysées par Pagani, Högberg, Fernandez et Siracusano (2013), les résultats suggèrent une activation réduite des aires frontomédiales et frontolatérales après la psychothérapie EMDR. Ces investigations en neuroimagerie sur l'EMDR indiquent un déplacement de l'activation des régions limbiques sous-corticales vers les aires corticales de régulation cognitive et émotionnelle.

Il faut souligner que ces études n'ont pas examiné les effets aigus de la stimulation bilatérale pendant la contemplation de l'événement traumatique et du traitement associatif subséquent mais se sont davantage penchées sur les effets neurocognitifs à plus long terme de la psychothérapie EMDR. L'exception à ce jour est l'étude EEG sur l'EMDR au cours de trois à huit séances de psychothérapie réalisée Pagani et coll. (2012). Ces chercheurs ont aussi étudié les effets sur l'EEG pendant les SBL lors de la première et de la dernière séance. Ils ont montré une activation accrue lors de la première séance au niveau du cortex préfrontal rostral gauche et du cortex visuel droit, avec une activation accrue du gyrus fusiforme gauche suivant les SBL après plusieurs séances d'EMDR. Pagani et coll. interprètent ces résultats comme indiquant un changement depuis des associations émotionnelles autoréférencées accrues en début de psychothérapie vers une augmentation du traitement cognitif et de la régulation des affects traumatiques après une psychothérapie EMDR réussie.

\section{Le modèle de cohérence interhémisphérique $(\mathrm{Clh})$}

L'un des neuromécanismes proposés les plus testables et donc les plus populaires pour expliquer l'efficacité de l'EMDR a été le modèle de cohérence interhémisphérique (CIh) auquel Shapiro (1989) a fait allusion la première mais qui a été formellement proposé par Bergmann $(1998,2000)$ et Servan-Schreiber (2000), puis empiriquement élaboré par Christman et ses collègues (e.g., Propper \& Christman, 2008). Ce modèle suggère que la stimulation saccadique bilatérale en EMDR sollicite des réseaux neuraux des deux côtés du cerveau et permettent donc à ces réseaux jusque-là dissociés de se relier aux événements traumatiques ciblés, permettant ainsi un retraitement plus constructif de l'événement traumatique. Cette interconnectivité accrue conduirait hypothétiquement à une meilleure régulation corticale préfrontale, par le cortex cingulaire antérieur, de l'amygdale hyperréactive et à une diminution physiologique de l'activation dans le cerveau limbique. Un test de ce modèle de CIh devrait pouvoir démontrer (a) un meilleur rappel des souvenirs et (b) une meilleure connectivité interhémisphérique après la stimulation bilatérale. 
Christman et ses collègues ainsi que d'autres chercheurs indépendants ont démontré de manière tout à fait convaincante une amélioration du rappel de souvenirs après une stimulation bilatérale au travers de divers paradigmes (voir Propper \& Christman, 2008 pour une analyse de cette littérature). Cependant la démonstration d'une connectivité interhémisphérique renforcée après une stimulation bilatérale n’a pas été aussi convaincante.

Une mesure fréquemment utilisée de la connectivité fonctionnelle dans le cortex est la cohérence EEG. La cohérence EEG est la fonction de densité spectrale des ondes normalisée par les spectres de puissance (Nunez et coll., 1997). Les valeurs de cohérence sont équivalentes à une fonction de corrélation qui aurait une grandeur entre 0 et 61 . La cohérence peut donc s'interpréter comme la communication ou la connectivité fonctionnelle entre deux sites d'enregistrement, avec une cohérence plus élevée représentant une meilleure coopération et synchronisation pour une fréquence donnée entre les zones cérébrales mesurées (Knott, LaBelle, Jones \& Mahoney, 2002 ; Nunez et coll., 1997 ; Weiss \& Mueller, 2003).

À ce jour, trois études testant les effets des mouvements oculaires saccadiques horizontaux sur la cohérence interhémisphérique ont été publiées. Une étude de Propper, Pierce, Geisler, Christman et Bellorado (2007), n'employant qu'un seul jeu d'électrodes bilatéraux frontaux aux sites $\mathrm{Fp} 1$ et $\mathrm{Fp} 2$, a montré une cohérence réduite des fréquences gamma $(35-54 \mathrm{~Hz})$ en réponse à stimulus se déplaçant de manière bilatérale par rapport à la condition contrôle d'un point clignotant rouge/vert.

Bien que les auteurs aient interprété leurs résultats comme indiquant des changements de cohérence interhémisphérique induits par les mouvements oculaires, on peut considérer qu'ils possèdent une pertinence discutable pour la pratique de l'EMDR et pour la connectivité interhémisphérique associée à une tâche pour les raisons suivantes : leur recours à des sites d'électrodes très sensibles aux artéfacts résiduels induits par les mouvements oculaires, un effet plafond des valeurs de cohérence alpha et thêta qui réduisent la probabilité d'obtenir des augmentations importantes et significatives pour ces fréquences et l'absence d'une tâche de rappel d'un souvenir épisodique pendant les mouvements oculaires.

La deuxième étude de Samara et coll. (2011) a calculé la cohérence de phase et d'amplitude par une EEG complète avant et après la participation à une tâche de rappel de mots neutres et émotionnels, avec les mêmes conditions MOB et contrôle que Propper et coll. (2007). Toutefois, elle a utilisé un plan intrasujets plus puissant, des électro-oculogrammes pour vérifier les $\mathrm{MOB}$ et une suppression visuelle et statistique laborieuse des artéfacts musculaires et de bruit. Avec ces conditions de contrôle minutieuses et de multiples analyses post-hoc pour trouver les effets de cohérence, Samara et coll. ont été incapables, en comparant l'avant- et l'après-MOB ou les différentes conditions de MO, de trouver un changement de cohérence EEG de phase ou d'amplitude constant ou prédit. Cependant, ils n'ont pas non plus utilisé de véritable de tâche de rappel d'un souvenir pendant les mouvements oculaires, qui constitue une partie essentielle de l'EMDR clinique, et ils ont inséré un film documentaire neutre de 30 minutes après la présentation de mots et avant l'enregistrement EEG et les MOB. Ainsi, en tant que test des effets EEG des MOB pendant le rappel de souvenirs épisodiques, cette étude ne présente pas de validité externe.

Jusqu'à ce que la cohérence soit mesurée pendant ou immédiatement après des stimulations bilatérales alors que le participant contemple des souvenirs épisodiques personnels significatifs, l'hypothèse CIh pour expliquer les effets de l'EMDR sur le retraitement des souvenirs traumatiques ne sera pas testé. C'est pourquoi nous avons proposé un test plus complet et une extension de l'hypothèse CIh. Keller, Stevens, Lui, Murray et Yaggie (2014) ont réalisé des EEG à 19 voies au cours de la phase d'association libre suivant la contemplation de souvenirs épisodiques positifs chez 30 participants non cliniques dans une de trois conditions de focalisation : un point fixe, un point clignotant rouge/vert ou des MOB saccadiques. Les résultats n'ont pas soutenu un modèle CIh des MOB mais ont obtenu des augmentations significatives au niveau de la cohérence interhémisphérique EEG des ondes delta et bêta basses dans les aires de traitement exécutif frontales droites et gauches. Ces auteurs ont proposé une élaboration du modèle de connectivité CIh, initialement proposé par Bergmann (1998, 2000) et Servan-Schreiber (2000), vers un modèle de cohérence corticale.

Dans cette modification, le niveau de mobilisation de centres neurologiques éloignés, reflétés par la cohérence corticale régionale spécifique, se produit en fonction de l'envergure du traitement de l'événement indexé. Elle offre deux prédictions fondées sur la nature du souvenir traité : (a) le traitement de souvenirs moins traumatiques peut se produire plus localement et de manière plus régionale, ce qui sera reflété par une cohérence EEG intrahémisphérique renforcée et (b) le traitement de souvenirs désagréables et traumatiques plus émotionnellement et cognitivement complexes pourrait faire appel à des connexions à des 
centres neurologiques plus éloignés et impliquer une cohérence EEG interhémisphérique renforcée ainsi qu'une cohérence intrahémisphérique accrue. Cette hypothèse pourrait être facilement testée dans une reproduction de l'étude de Keller et coll. (2014) qui utilise le même paradigme fondamental mais le traitement d'un souvenir désagréable. Cette étude de suivi est présentée dans ce projet de recherche.

\section{Méthodes}

\section{Participants}

Les participants étaient constitués de 46 étudiantes de premier cycle d'une université du Sud-Ouest américain, recrutées en tant que volontaires non cliniques du groupe de sujets de la faculté de psychologie. Des tests précédents au sein du groupe avaient identifié des participantes potentielles qui étaient droitières (Edinburgh Handedness Inventory ; Oldfield, 1971) ; montraient peu de tendances dissociatives (score inférieur à 30 sur l'échelle des expériences dissociatives [Dissociative Experience Scale] de Carlson \& Putnam [1993]) ; et répondaient aux critères d'inclusion d'absence de grossesse actuelle, d'antécédent de trauma crânien, de perte de connaissance, d'épilepsie et de prise de médicament sur ordonnance ou de drogue. L'âge moyen était de 20,33 ans ( $\sigma=2,68$ ans) et il n'y avait pas de différence d'âge significative entre les trois conditions de traitement, $F(2,43)=0,324, p=0,725$. Chaque participante était affectée de manière aléatoire à l'une des trois conditions de traitement dans ce plan mixte inter/intra-groupes. Toutes les participantes ont reçu des crédits de formation pour leur participation à cette étude qui a été approuvée par le comité de protection des personnes de l'université de l'Arizona du Nord.

\section{Instruments}

En tant que mesure de référence avant la participation aux conditions de traitement, chaque participante sélectionnée a évalué son événement cible désagréable identifié sur l'échelle d'impact de l'événement (Impact of Event Scale [IES] : Horowitz, Wilner \& Alvarez, 1979). Chaque participante devait obtenir un score correspondant au niveau modéré de détresse (9-19) sur l'IES pour l'événement désagréable identifié. Les participantes ont également évalué leur événement cible sur l'échelle visuelle analogique en 11 points de 0 à 10 [où $10=$ hautement perturbé(e)] Subjective Units of Discomfort (unités subjectives d'inconfort ; Lee \& Drummond, 2008), sur l'échelle visuelle analogique en 11 points (où $10=$ extrêmement vive) Image
Vividness Scale (échelle de vivacité de l'image [IVS]), et sur l'échelle visuelle analogique en 21 points (de -10 à +10 , où $-10=$ extrêmement désagréable) Emotional Valence Scale (échelle de valence émotionnelle [EVS]; Aitken, 1969). Les mesures des échelles IES et SUD n'étaient utilisées que dans la sélection d'un événement cible désagréable approprié ; l'IVS et l'EVS étaient utilisées pour évaluer le changement de vivacité et de valence émotionnelle du stimulus cible.

Les évaluations IVS et EVS ont été réalisées à six occasions : avant chaque condition stimulus initiale et après chacune des cinq périodes d'une minute d'association libre qui suivaient chaque condition de mouvements oculaires de 24 secondes.

Dans le but de la meilleure cohérence possible, tant à l'égard du protocole EMDR qu'entre participantes, les stimulations visuelles étaient apportées par une barre lumineuse EyeScan 2000S Light Bar (1994) conçue pour un usage clinique en EMDR. Les saccades bilatérales consistaient en une saccade gauche-droite ou droite-gauche toutes les 500 millisecondes, produisant deux mouvements oculaires par seconde, pendant un total de 24 secondes. La barre lumineuse était positionnée à la hauteur des yeux à une distance de $35 \mathrm{~cm}$ (environ 14 pouces) de la participante. La fixation d'un point immobile (POINT) était réalisée en demandant à la participante de fixer son regard sur la lumière centrale fixe de la barre lumineuse et ne pas bouger les yeux ou la tête. La condition de fixation du point immobile avec mouvement bilatéral en arrière-plan d'une lumière (MAP) était créée en demandant à la participante de fixer son regard sur le centre de la barre lumineuse et de ne pas bouger ses yeux pendant que les lumières se déplaçaient bilatéralement. (Cette condition contrôle remplace la condition de point clignotant rouge/vert utilisée dans notre précédente étude [Keller et coll., 2014] et par Christman, Propper \& Dion [2004] en raison des effets directs observés de cette condition sur la cohérence des basses fréquences [Keller et coll., 2014] et en vue d'examiner si les MOB eux-mêmes, plutôt que les stimulations visuelles bilatérales, jouent un rôle critique dans les effets de cohérence.) La condition MOB était réalisée en demandant à la participante de suivre des yeux la lumière se déplaçant de manière bilatérale sur la barre lumineuse EMDR, sans bouger sa tête.

Les données EEG étaient enregistrées à l'aide d'un système d'acquisition EEG à 24 voies Mitsar (2000) avec un taux d'acquisition des signaux de $500 \mathrm{~Hz}$ et une impédance d'entrée de l'amplificateur d'au moins 200 Mohms. Les données EEG à 19 voies 
étaient recueillies à l'aide du logiciel WinEEG (2000) avec un taux d'échantillonnage de conversion $\mathrm{A} / \mathrm{D}$ de $256 \mathrm{~Hz}$, des filtres passe-haut et passe-bas de 0,3 $\mathrm{Hz}$ et $50 \mathrm{~Hz}$ respectivement, et un filtre coupe-bande de $60 \mathrm{~Hz}$. Les données étaient analysées et artéfactées à l'aide des logiciels de traitement et d'analyse de données NovaTech EEG Eureka! (2000) et MHyT (2000). Le système international 10-20 de placement des électrodes EEG a été utilisé pour le placement des 19 électrodes monopolaires $\mathrm{Ag} / \mathrm{AgCl}$ sur le cuir chevelu à l'aide du système Electro Cap (1983) avec des électrodes de référence de l'oreille mathématiquement liés. Les impédances des électrodes étaient réglées à moins de $5 \mathrm{kohms}$ et pour ne pas différer entre elles de plus d'un kohm. Les données EEG brutes étaient artéfactées visuellement à deux reprises par deux artéfacteurs formés et indépendants, aveugles aux conditions de traitement, à l'aide de critères exigeants et rédigés avec précision, pour supprimer l'EMG et les autres artéfacts de bruit. Un logiciel d'analyse EEG était employé pour réaliser des transformations de Fourier rapides FFT) et des analyses de puissance spectrale et de cohérence sur les données brutes. Vbmapper (Frederick, 2001) a été utilisé pour créer des cartes de cohérences bidimensionnelles des changements de cohérence pré- à post-traitement significatifs. L'analyse FFT employait le rétrécissement du domaine temporel de Hamming, le lissage du domaine des fréquences de Blackman, un facteur d'avancement des fenêtres FFT superposées égal à 8 , et un filtre de lissage de la moyenne mobile égale à 3 . Cinq bandes de fréquences étaient examinées dans ces analyses : delta (1-3,99 Hz), thêta $(4-7,99 \mathrm{~Hz})$, alpha $(8-11,99 \mathrm{~Hz})$, bêta $(12-29,99 \mathrm{~Hz})$ et gamma $(30-50 \mathrm{~Hz})$.

\section{Procédure}

La portion de l'étude portant sur la cohérence EEG se déroulait entre 13 heures et 18 heures pour contrôler les variations diurnes des niveaux des hormones du stress, particulièrement le cortisol, susceptibles d'affecter de manière différentielle les relevés EEG (Barenne \& Gibbs, 1942 ; Brown, 1994). De plus, nos participantes n'étaient testées que pendant la phase lutéale de leur cycle menstruel pour contrôler plus avant les différences documentées de différences de mesures EEG en fonction des phases menstruelles (Barenne \& Gibbs, 1942 ; Kaplan, Whitsett \& Robinson, 1990 ; Solis-Oritz, Ramos, Arce, Guevara \& Corsi-Cabera, 1994).

Le jour de l'EEG, les participantes donnaient leur consentement éclairé puis étaient affectées de manière aléatoire à l'une des trois conditions de traitement. Les participantes étaient confortablement assises et droites dans un fauteuil inclinable et toutes les données étaient enregistrées dans des locaux de recherche phoniquement isolés. Un miroir quadrillé d'environ $20 \mathrm{~cm}$ était positionné sur le mur faisant face à la patiente, placé de biais, de sorte que le chercheur puisse observer la présence de mouvements oculaires et établir si les yeux étaient ouverts ou fermés, sans que la participante ne puisse observer son reflet dans le miroir. Des consignes standardisées étaient présentées à l'aide de $\mathrm{CD}$ préenregistrés distincts pour chaque condition. Le casque EEG à 19 électrodes était placé sur la tête de la participante, les électrodes étaient activées et un tracé EEG clair a pu être établi. Un relevé EEG de référence avec les yeux fixes a été enregistré pendant 5 minutes au cours desquelles la participante était invité à se détendre et « à vider son esprit puis à suivre toute pensée qui survient».

On demandait aux participantes de se rappeler et de décrire à l'expérimentateur un événement désagréable qui leur avait occasionné une gêne émotionnelle modérée et qu'elles se rappelaient clairement. Le souvenir choisi était évalué entre 6 et 8 sur la sous-échelle de détresse SUD. Si la participante ne se rappelait pas d'événement particulier, quelques exemples étaient proposés comme échouer à un examen ou manquer une échéance à l'école, tomber d'un vélo dans l'enfance ou une rupture amoureuse. On leur disait de se rappeler l'événement (qui était présent, où cela s'est produit, etc.), avec un instantané visuel de l'événement, les pensées négatives le concernant et les émotions et les sensations impliquées dans le souvenir. Les participantes évaluaient alors le souvenir désagréable sur l'échelle SUD. Un événement n'était pas utilisé s'il était trop perturbant (9 ou 10) ou ne provoquait pas suffisamment de détresse (0-5). Les participantes remplissaient ensuite l'IES en rapport avec cet événement. Tous les souvenirs désagréables qui rencontraient les critères de l'échelle SUD répondaient également à ceux de l'IES, ainsi aucune participante n'a été perdue dans ce processus. Ensuite les participantes étaient invitées à se rappeler le souvenir désagréable et à en évaluer la vivacité et l'intensité émotionnelle sur l'IVS et l'EVS respectivement.

L'une des trois conditions de traitement était alors présentée à chaque participante. La participante se rappelait l'événement déplaisant dans les détails déjà décrits tout en se prêtant à la condition de mouvements oculaires à laquelle elle était affectée. Après 24 secondes, la participante devait fermer les yeux 
et vider son esprit, puis ouvrir les yeux et regarder devant elle pendant 60 secondes " en suivant toute pensée qui vient ». Des EEG continus étaient enregistrés pendant cette période d'association libre de 60 secondes. On demandait ensuite à la participante de se rappeler le souvenir initial et d'évaluer sa vivacité et la valence émotionnelle associée sur les échelles IVS et EVS. Le chercheur observait les yeux de la participante dans le miroir pour vérifier que le regard demeurait fixe pendant la contemplation du souvenir épisodique.

Dans la condition de fixation d'un point immobile (Point), chaque participante $(\mathrm{n}=15)$ était invitée à se rappeler l'événement désagréable en détail en fixant le point lumineux central de la barre lumineuse EMDR pendant 24 secondes. La condition Point a donc fourni des tracés de la cohérence EEG pour un stimulus contrôle sans stimulation bilatérale.

Dans la condition des yeux fixes avec un point se déplaçant de manière bilatérale en arrière-plan (MAP), chaque participante $(n=16)$ était invitée à se rappeler l'événement désagréable en détail en fixant le centre de la barre lumineuse sans bouger les yeux pendant 24 secondes. Cette condition a fourni les tracés EEG suivant la fixation oculaire avec des mouvements en arrière-plan du champ visuel pendant la contemplation d'un souvenir épisodique négatif pour mieux déterminer si le mouvements oculaires physiques sont réellement nécessaires à la cohérence accrue prédite.

Dans la condition $M O B$, chaque participante $(n=$ 15) était invitée à se rappeler l'événement désagréable en détail en suivant des yeux et sans bouger la tête, un point lumineux se déplaçant de manière bilatérale sur une barre lumineuse EMDR. Cette condition a fourni les enregistrements EEG suivant des MOB pendant la contemplation d'un souvenir épisodique négatif pour tester l'hypothèse d'une cohérence accrue après les MOB.

Chaque condition était répétée cinq fois pour chaque participante. Les enregistrements EEG étaient donc réalisés pendant un total de 5 minutes après les conditions stimuli. À la fin de la procédure, on débranchait l'équipement et on échangeait brièvement avec les participantes qui pouvaient ensuite quitter le laboratoire. Cet échange couvrait des informations sur des services de soutien psychologique le cas échéant après la procédure. Pour renforcer la validité externe de l'étude, la procédure MOB reproduisait autant que possible le schéma de la phase 4 en EMDR (protocole de désensibilisation), les enregistrements EEG se déroulant pendant la période d'association libre juste après les $\mathrm{MOB}$ en se rappelant le souvenir cible. Et bien que cette étude n’ait pas fait appel à des individus atteints d'ESPT, les participantes se rappelaient les événements déplaisants en détail de manière similaire aux procédures employées dans des études antérieures (Lee $\&$ Drummond, 2008 ; Van den Hout, Muris, Salemink \& Kindt, 2001).

\section{Plan et analyse}

Cette étude employait un plan expérimental mixte inter-/intragroupes avec la condition de participation en tant que variable inter- et le moment de la mesure comme variable intra- ${ }^{-1}$. Une ANOVA à mesures répétées était utilisée dans l'analyse des variables vivacité et valence émotionnelle pour les cinq points de mesure. En raison du faible nombre de participantes dans chaque groupe et des différences entre les groupes au niveau des mesures EEG de référence, une ANCOVA intergroupes était employée pour les analyses de cohérence. Cette analyse EEG prenait en compte deux niveaux.

Pour l'analyse principale, les valeurs de la cohérence prétraitement pour la fréquence EEG et l'aire corticale respectives étaient saisies en tant que covariables dans des analyses ANCOVA unidirectionnelles distinctes. Cette analyse permettait d'examiner les différences de groupe post-traitement, la variabilité des valeurs de référence étant contrôlée. Si les résultats étaient significatifs, alors des comparaisons des effets simples par test $t$ étaient effectuées pour déterminer à quel niveau se produisaient les différences significatives. Pour l'analyse secondaire, les changements prétraitement à post-traitement des groupes montrant des effets significatifs dans l'analyse principale étaient alors évalués. Ces analyses de statistique inférentielle étaient rapportées puis suivies de statistiques descriptives pour présenter visuellement les résultats obtenus.

Après avoir artéfacté les mesures de référence de 5 minutes et les enregistrements EEG post-stimulus cumulés de 5 minutes, une moyenne de 4,03 minutes $(\sigma=0,48)$ pour les valeurs de référence et une moyenne de 4,14 minutes $(\sigma=0,75)$ pour les données EEG post-stimulus ont été obtenues. Les valeurs de cohérence entre toutes les paires d'électrodes possibles pour chaque fréquence EEG désignée étaient générées par Eureka! et traitées à l'aide des mêmes procédures algorithmiques que celles employées par Keller et coll. (2014).

Des cartographies de la cohérence entre des paires d'électrodes interconnectées pour les fréquences significatives ont également été générées pour mieux situer et afficher les régions corticales possédant des changements de cohérence. Pour créer ces 


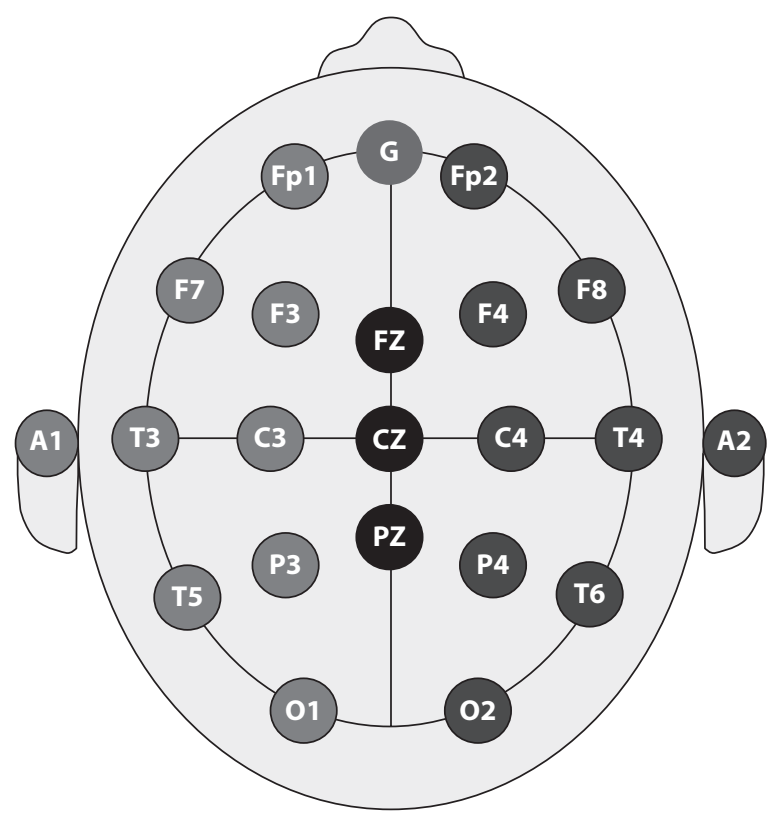

FIGURE 1. Schéma du système international 10-20 pour le placement d'électrodes EEG.

cartographies, les valeurs de cohérence pour les paires d'électrodes au sein de groupes d'électrodes identifiés et pour les périodes pré- et post-traitement ont été saisies dans le logiciel de cartographie de la cohérence open-source Vbmapper (Frederick, 2001). Des cartographies étaient alors générées pour les différences de cohérence de paires d'électrodes au sein de groupes lorsqu'elles atteignaient un niveau de signification statistique.

Pour simplifier l'analyse par des régions fonctionnelles interhémisphériques larges, nous avons sélectionné des paires d'électrodes homologues dans chaque hémisphère en les regroupant par régions frontales (la moyenne de Fp1-Fp2, F3-F4, F7-F8), centrales (C3-C4), pariétales (P3-P4), temporales (la moyenne de T3-T4, T5-T6) et occipitales (O1-O2) pour obtenir cinq valeurs de cohérence EEG régionale pour chaque bande de fréquences (voir Figure 1). Pour la cohérence intrahémisphérique, nous avons examiné, pour chaque fréquence EEG, les groupes de cohérence intrahémisphérique régionale frontale gauche (Fz-Fp1, Fz-F3, Fz-F7) et frontale droite (Fz-Fp2, Fz-F4, Fz-F8), centrale gauche $(\mathrm{Cz}-\mathrm{C} 3$, $\mathrm{Cz}-\mathrm{T} 3)$ et centrale droite $(\mathrm{Cz}-\mathrm{C} 4, \mathrm{Cz}-\mathrm{T} 4)$, pariétale gauche $(\mathrm{Pz}-\mathrm{P} 3, \mathrm{Pz}-\mathrm{T} 5)$ et pariétale droite $(\mathrm{Pz}-\mathrm{P} 4$, $\mathrm{Pz}-\mathrm{T} 6)$, et occipitale gauche (Pz-O1) et occipitale droite (Pz-O2). La moyenne des valeurs de la cohérence au sein de chaque groupe régional (frontal, central, pariétal) était calculée. Ces groupes de données interhémisphériques et intrahémisphériques étaient alors examinés pour la normalité et l'homogénéité des hypothèses de variance ; ils ont vérifié les hypothèses en vue d'une analyse paramétrique supplémentaire.

Comme il s'agissait d'une étude d'investigation sur un échantillon relativement réduit (15 à 16 participantes par cellule) avec des comparaisons planifiées et qu'il n'y avait pas plus de comparaisons que de degrés de liberté pour l'effet de chaque analyse d'EEG distincte, aucun ajustement n'a été requis pour l'inflation du taux d'erreur famille (Tabachnick \& Fidell, 2013). Puisque ces dernières conditions n'étaient pas remplies pour les comparaisons entre conditions pour la vivacité de l'image et la valence émotionnelle du souvenir, ces analyses ont été ajustées avec la méthode de Bonferroni. Le seuil de signification alpha a été fixé à 0,05 .

\section{Résultats}

Les effets des conditions sur la vivacité de l'image désagréable et de la valence émotionnelle du souvenir

Dans les trois conditions, des changements statistiquement significatifs (avec la correction des ddl de Greenhouse-Geisser) ont été observés au niveau de la vivacité et de la valence émotionnelle du souvenir initial après la condition de présentation. Plus précisément, la vivacité de l'image diminuait significativement dans tous les essais $F(3,22 ; 138,55)=14,029$, $p<0,0001, \eta^{2}=0,246$, sans effets significatifs de la condition, $F(2 ; 43)=2,240, p=0,119, \eta^{2}=0,094$ ou de l'interaction $F(6,44 ; 138,55)=0,756, p=0,615$, $\eta^{2}=0,034$. Les comparaisons planifiées entre les conditions pour chaque présentation de l'essai n'ont révélé aucune différence significative entre les conditions pour le prétraitement ou pour les essais $(p<0,05)$, à l'exception de l'essai 3 pour lequel la condition MOB a montré une vivacité de l'image significativement plus importante que les conditions MAP, $t(29)=2,625$, $p=0,007$, et Point, $t(28)=2,103, p=0,023$. Les changements pour chaque condition et pour tous les essais sont présentés dans la Figure 2.

En ce qui concerne la valence émotionnelle du souvenir désagréable, des diminutions statistiquement significatives (avec la correction des ddl de Greenhouse-Geisser) du caractère déplaisant des événements négatifs identifiés étaient observées pour tous les essais, $F(2,23 ; 95,96)=12,642, p<0,0001$, $\eta^{2}=0,227$, sans effet significatif de la condition, $F(2 ; 43)=0,237, p=0,790, \eta^{2}=0,11$, ni de l'interaction, $F(4,46 ; 95,96)=0,694, p=0,614, \eta^{2}=0,031$. Les comparaisons planifiées entre les conditions pour 


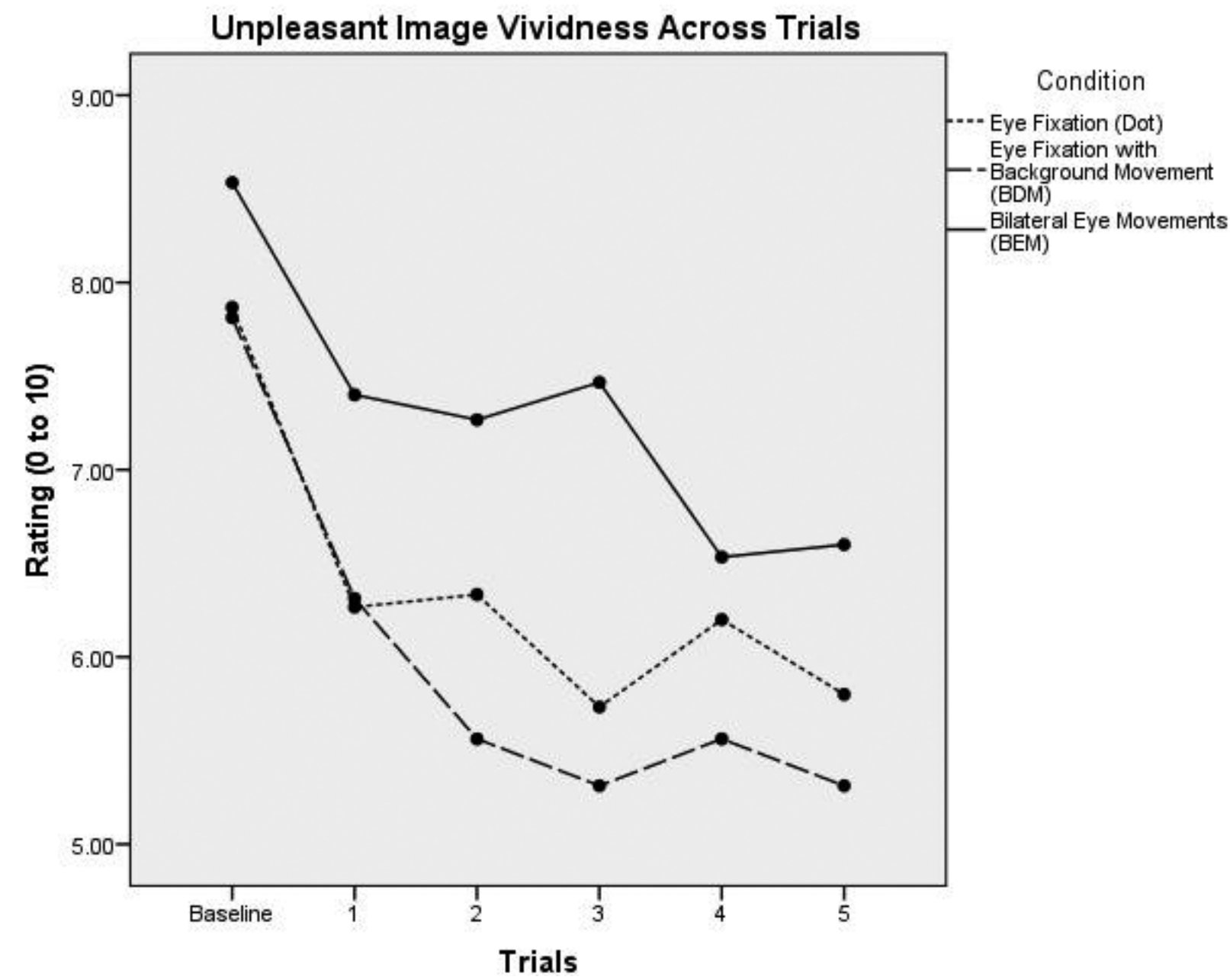

FIGURE 2. Niveaux de vivacité de l'image désagréable pour tous les essais d'enregistrement EEG et pour chaque condition. La vivacité de l'image est évaluée entre 0 et 10 , où $10=$ extrêmement vive.

Note. Unpleasant Image Vividness Across Trials = vivacité de l'image désagréable pour les différents essais ; Rating $(0$ to 10$)=$ niveau $($ de 0 à 10$)$; Condition $=$ condition $;$ Eye Fixation $($ Dot $)=$ Yeux fixes (Point) ; Eye Fixation with Backgound Movement $(\mathrm{BDM})=$ yeux fixes avec un mouvement en arrière-plan (MAP); Bilateral Eye Movements $(\mathrm{BEM})=$ mouvements oculaires bilatéraux $(\mathrm{MOB}) ;$ Baseline $=$ valeurs de référence $;$ Trials $=$ essais.

chaque présentation de l'essai n’ont révélé aucune différence significative entre les conditions pour le prétraitement ou pour les essais $(p<0,05)$. Les changements pour chaque condition et pour tous les essais sont présentés dans la Figure 3.

Toutefois, l'examen de la Figure 3 révèle une tendance différentielle à des changements au niveau de la valence émotionnelle pour la condition MOB par rapport aux deux conditions de contrôle suivant la participation à une condition. Des comparaisons de tests $t$ entre essais successifs pour chaque condition soutiennent cette observation d'une tendance assez remarquable visuellement mais non significative vers une diminution de la valence émotionnelle négative entre l'essai 2 et l'essai 3 pour la condition $\mathrm{MOB}$ seulement, $t(14)=21,741, p=0,052$.

\section{Effets de cohérence interhémisphérique}

Cohérence bêta frontale. Les analyses ANCOVA principales des effets de cohérence interhémisphérique pour chaque aire corticale identifiée et pour chaque bande de fréquences EEG, avec les valeurs de cohérence de référence respectives en tant que covariables, ont révélé des effets principaux significatifs de la condition pour la cohérence bêta frontale, $F(2$, 42) $=3,488, p=0,040, \eta^{2}=0,142$. Les comparaisons planifiées d'effets simples ont montré que la condition MOB était significativement plus élevée en cohérence que la condition Point après la participation à la condition, $t(28)=2,32, p=0,024$. Les comparaisons secondaires test $t$ des groupes dépendants évaluant les changements avant et après la participation à chacune 


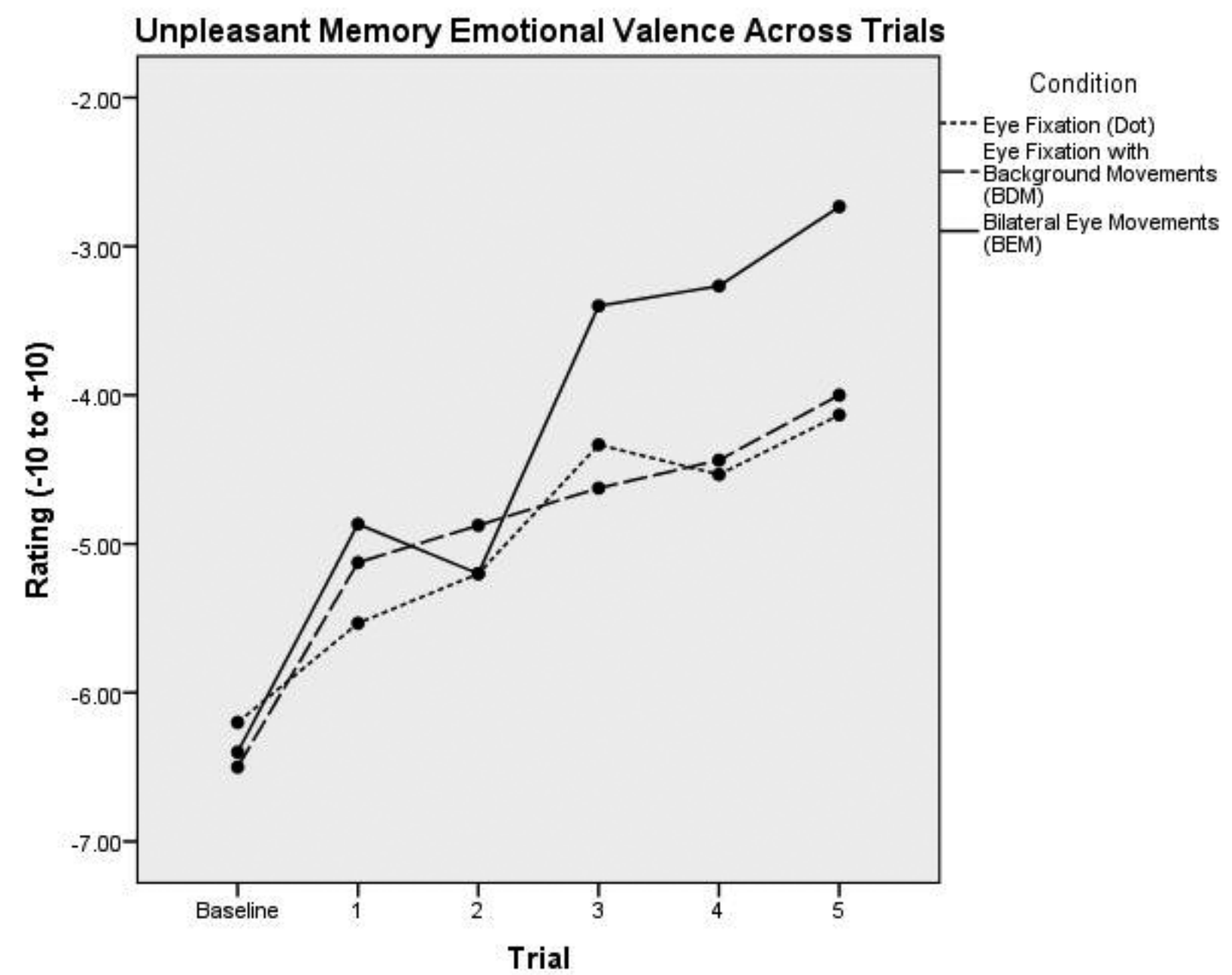

FIGURE 3. Niveaux de valence émotionnelle du souvenir désagréable pour les différents essais d'enregistrement EEG pour chaque condition. La valence émotionnelle est évaluée entre -10 et +10 , où $-10=$ extrêmement désagréable.

Note. Unpleasant Memory Emotional Valence Across Trials = valence émotionnelle de souvenirs désagréables pour les différents essais ; Rating (0 to 10) = niveau (de 0 à 10); Condition $=$ condition ; Eye Fixation (Dot) $=$ Yeux fixes (Point) ; Eye Fixation with Backgound Movement $(B D M)=$ yeux fixes avec un mouvement en arrière-plan (MAP) ; Bilateral Eye Movements $(\mathrm{BEM})=$ mouvements oculaires bilatéraux $(\mathrm{MOB}) ;$ Baseline $=$ valeurs de référence ; Trials $=$ essais.

des conditions pour la cohérence bêta frontale ont indiqué des diminutions statistiquement significatives de la cohérence Point, $t(14)=2,433, p=0,015$. Il y avait une tendance non significative de la cohérence bêta frontale MOB à augmenter après la participation à la condition, $t(14)=21,646, p=0,061$.

Cohérence gamma frontale. Seule une tendance s'approchant de la signification a été obtenue pour la cohérence gamma frontale, $F(2,42)=2,433$, $p=0,100, \eta^{2}=0,104$ dans l'analyse principale. Pour évaluer plus avant cet effet hypothétique, des comparaisons planifiées des effets simples ont été réalisées, indiquant que la condition $\mathrm{MOB}$ était significativement plus élevée en cohérence que la condition Point, $t(28)=2,10, p=0,038$. Des comparaisons test $t$ secondaires des groups dépendants évaluant les changements de cohérence avant et après la participation à chacune des conditions pour la cohérence gamma frontale ont révélé des diminutions statistiquement significatives pour la condition Point, $t(14)=2,936, p=0,006$. Aucune autre comparaison de conditions interhémisphériques n’a atteint de signification statistique, quelles que soient l'aire ou la fréquence.

\section{Effets de cohérence intrahémisphérique}

L'analyse ANCOVA de la cohérence intrahémisphérique pour chaque fréquence et chaque aire corticale a apporté plusieurs résultats significatifs. Tous les effets statistiques de cohérence intrahémisphérique pour les fréquences EEG thêta, bêta et gamma sont présentés dans le Tableau 1. Aucune autre fréquence ni aire corticale n'a atteint de signification statistique pour quelque condition que ce soit ${ }^{2}$. 
TABLEAU 1. Moyennes des cohérences et résultats des test t pré- et post-traitement pour les effets de cohérence intrahémisphérique frontale thêta, bêta et gamma

\begin{tabular}{|c|c|c|c|c|}
\hline Condition/fréquence/hémisphère & $\begin{array}{l}\text { Moyenne pré- } \\
\text { traitement } \\
\text { (écart-type) }\end{array}$ & $\begin{array}{l}\text { Moyenne post- } \\
\text { traitement } \\
\text { (écart-type) }\end{array}$ & $\begin{array}{c}\text { Valeur } \\
\text { de } t\end{array}$ & $\begin{array}{l}\text { Valeur } \\
\text { de } p\end{array}$ \\
\hline \multicolumn{5}{|l|}{ Yeux fixes (Point) } \\
\hline Thêta frontal gauche & $0,651(0,019)$ & $0,663(0,091)$ & $-2,770$ & $0,008^{\star \star \star}$ \\
\hline Thêta frontal droit & $0,655(0,016)$ & $0,659(0,015)$ & $-1,235$ & 0,119 \\
\hline \multicolumn{5}{|c|}{ Yeux fixes avec mouvements en arrière-plan (MAP) } \\
\hline Thêta frontal gauche & $0,669(0,019)$ & $0,669(0,019)$ & $-0,163$ & 0,437 \\
\hline Thêta frontal droit & $0,675(0,011)$ & $0,677(0,010)$ & $-0,382$ & 0,354 \\
\hline \multicolumn{5}{|c|}{ Mouvements oculaires bilatéraux (MOB) } \\
\hline Thêta frontal gauche & $0,671(0,021)$ & $0,692(0,015)$ & $-1,748$ & $0,051^{\star}$ \\
\hline Thêta frontal droit & $0,675(0,017)$ & $0,692(0,015)$ & $-2,620$ & $0,010^{\star \star \star}$ \\
\hline \multicolumn{5}{|l|}{ Yeux fixes (Point) } \\
\hline Bêta frontal gauche & $0,442(0,049)$ & $0,394(0,043)$ & 2,014 & $0,032^{\star \star}$ \\
\hline Bêta frontal droit & $0,436(0,043)$ & $0,394(0,035)$ & 2,029 & $0,031^{\star \star}$ \\
\hline \multicolumn{5}{|c|}{ Yeux fixes avec mouvements en arrière-plan (MAP) } \\
\hline Bêta frontal gauche & $0,469(0,033)$ & $0,448(0,039)$ & 0,710 & 0,245 \\
\hline Bêta frontal droit & $0,468(0,032)$ & $0,443(0,039)$ & 0,711 & 0,244 \\
\hline \multicolumn{5}{|c|}{ Mouvements oculaires bilatéraux (MOB) } \\
\hline Bêta frontal gauche & $0,456(0,048)$ & $0,497(0,041)$ & $-1,721$ & $0,054^{\star}$ \\
\hline Bêta frontal droit & $0,460(0,048)$ & $0,501(0,043)$ & $-2,072$ & $0,029^{\star \star}$ \\
\hline \multicolumn{5}{|l|}{ Yeux fixes (point) } \\
\hline Gamma frontal gauche & $0,332(0,039)$ & $0,276(0,033)$ & 2,522 & $0,012^{\star \star}$ \\
\hline Gamma frontal droit & $0,321(0,034)$ & $0,272(0,024)$ & 2,431 & $0,015^{\star \star}$ \\
\hline \multicolumn{5}{|c|}{ Yeux fixes avec mouvements en arrière-plan (MAP) } \\
\hline Gamma frontal gauche & $0,353(0,038)$ & $0,325(0,036)$ & 0,854 & 0,203 \\
\hline Gamma frontal droit & $0,340(0,035)$ & $0,307(0,033)$ & 0,900 & 0,192 \\
\hline \multicolumn{5}{|c|}{ Mouvements oculaires bilatéraux (MOB) } \\
\hline Gamma frontal gauche & $0,354(0,049)$ & $0,378(0,038)$ & $-0,938$ & 0,182 \\
\hline Gamma frontal droit & $0,338(0,050)$ & $0,377(0,042)$ & $-1,563$ & $0,070^{\star}$ \\
\hline
\end{tabular}

Note. Tous les tests sont des tests $t$ unilatéraux d'échantillons appariés avec ddl $=14$ pour Point et MOB et ddl $=15$ pour MAP. Une valeur négative du test $t$ indique une augmentation entre la cohérence prétraitement et la cohérence post-traitement.

${ }^{\star} p \leq 0,10^{\star \star} p \leq 0,05^{\star \star \star} p \leq 0,01$

Cohérence thêta frontale. Dans l'analyse principale, un effet de condition significatif a été trouvé pour les fréquences thêta frontales droites, $F(2,42)$ $=4,238, p=0,021, \eta^{2}=0,168$ avec une cohérence dans la condition MOB significativement supérieure à celle de la condition Point, $t(28)=2,50, p=0,018$, et à celle de la condition MAP, $t(29)=2,67, p=$ 0,013 . Des comparaisons secondaires test $t$ des groups dépendants pour les changements pré-post pour chaque condition ont révélé une augmentation signi- ficative de la cohérence MOB, $t(14)=22,620, p=$ 0,010 , seulement, sans changement significatif pour les cohérences Point, $t(14)=21,235, p=0,119$ ou MAP, $t(15)=20,382, p=0,354$.

Cohérence bêta frontale. De plus, un effet de condition statistiquement significatif a été trouvé pour les fréquences bêta frontales droites et gauches, respectivement, $F(2,42)=3,367, p=0,044, \eta^{2}=$ 0,138 et $F(2,42)=3,601, p=0,036, \eta^{2}=0,146$. 
Les comparaisons planifiées des effets simples ont déterminé que la cohérence MOB était significativement plus élevée que la cohérence Point pour les deux aires intrahémisphériques, $t(28)=2,50, p=0,016$ et $t(28)=2,66, p=0,011$, respectivement.

Les comparaisons test $t$ de suivi des groupes dépendants pour les changements pré/post ont révélé, pour la cohérence bêta frontale droite, une augmentation significative de la cohérence MOB, $t(14)=22,072$, $p=0,029$, avec une diminution significative de la cohérence Point, $t(14)=2,029, p=0,031$, et pas de changement significatif de la cohérence MAP, $t(15)=$ $0,711, p=0,244$. De manière similaire, pour la cohérence bêta frontale gauche, il y avait une diminution significative de la cohérence Point, $t(14)=2,014, p$ $=0,032$; une augmentation non significative de la cohérence MOB, $t(14)=21,721, p=0,054$; et pas de changement significatif pour la cohérence MAP, $t(15)=0,710, p=0,245$. Ces effets sur la cohérence avant et après la condition pour les fréquences bêta frontales droites et gauches sont présentés dans la Figure 4.

Cohérence gamma frontale. Un effet de la condition statistiquement significatif a été obtenu pour les cohérences EEG gamma frontales droite et gauche ainsi que dans les analyses principales, $F(2,42)=$ 4,109, $p=0,023, \eta^{2}=0,164$ et $F(2,42)=3,448, p$ $=0,041, \eta^{2}=0,141$ respectivement. Des comparaisons planifiées des effets simples ont montré que la cohérence MOB était significativement plus élevée que celle de Point pour les deux régions intrahémisphériques, $t(28)=2,76, p=0,009$ et $t(28)=2,56, p$ $=0,013$, respectivement. La cohérence frontale MOB était également significativement plus élevée que la cohérence MAP, $t(29)=2,06, p=0,042$, mais il n'y avait pas d'effet significatif relatif aux différences de cohérence frontale gauche MOB-MAP, $t(29)=1,58$, $p=0,119$.

Des comparaisons test $t$ secondaires des groups dépendants pour les changements pré/post ont révélé, pour la gamma frontale droite, une diminution significative de la cohérence Point, $t(14)=2,431, p=0,015$; une augmentation non significative de la cohérence MOB, $t(14)=21,563, p=0,070$; et pas de changement significatif de la cohérence MAP, $t(15)=0,900$, $p=0,192$. Pour la gamma frontale gauche, il n'y avait pas de changement significatif de la cohérence MOB, $t(14)=20,938, p=0,182$ ou de la cohérence MAP, $t(15)=0,854, p=0,203$, mais une diminution significative de la cohérence Point, $t(14)=2,522, p=0,012$. Ces effets de cohérence avant et après la condition pour les gammas frontales droite et gauche sont présentés dans la Figure 5.
Localisation topographique des effets des MOB

La Figure 6 correspond à une carte topographique des cohérences pour les changements de la fréquence EEG bêta entre le prétraitement et le post-traitement pour les MOB. Chaque ligne noire reliant une paire d'électrodes reflète une augmentation significative de la cohérence de cette paire tandis que les lignes grises représentent une cohérence significativement réduite. Les augmentations de la cohérence dans les localisations frontales et, de manière prédominante, hémisphériques droites sont clairement illustrées par cette carte pour les augmentations de cohérence de la fréquence bêta pendant les associations libres suivant les MOB en se rappelant le souvenir désagréable.

\section{Discussion}

En résumé, à la suite des associations libres avec des souvenirs désagréables auxquels les participantes prêtaient attention pendant les MOB, aucune augmentation significative de la cohérence interhémisphérique n’a été observée pour quelque fréquence EEG que ce soit dans cette étude. Pour les bandes bêta et gamma, des différences significatives ont été obtenues entre les cohérences MOB et Point après la participation à la condition. Cependant, ces différences pouvaient être attribuées à la diminution significative de la cohérence Point contrôle après la participation à la condition.

En ce qui concerne la cohérence intrahémisphérique toutefois, des augmentations statistiquement significatives dans les cohérences EEG thêta et bêta frontales droites suivant la condition MOB ont été observées. La cohérence MOB dans la région frontale droite était significativement plus élevée que les conditions Point contrôle et MAP pour les fréquences EEG thêta et gamma et plus élevée que Point pour la fréquence bêta suivant la participation à la condition. Les effets de la région frontale gauche suivait cette même répartition générale d'augmentations et de diminutions au cours du traitement mais sans augmentation significative de la cohérence intrahémisphérique MOB. Les diminutions de la cohérence Point pour les fréquences bêta et gamma dans ces régions frontales gauches semblent avoir pour résultat que la cohérence $\mathrm{MOB}$ soit significativement supérieure à la cohérence Point lors des mesures posttraitement. La cohérence intrahémisphérique thêta frontale gauche était significativement supérieure à la condition MAP de contrôle, apparemment en raison de la tendance à l'augmentation de la cohérence MOB au niveau de thêta avec le traitement. 
Left Frontal Beta Intrahemispheric Coherence Changes Across Trials for Each Condition

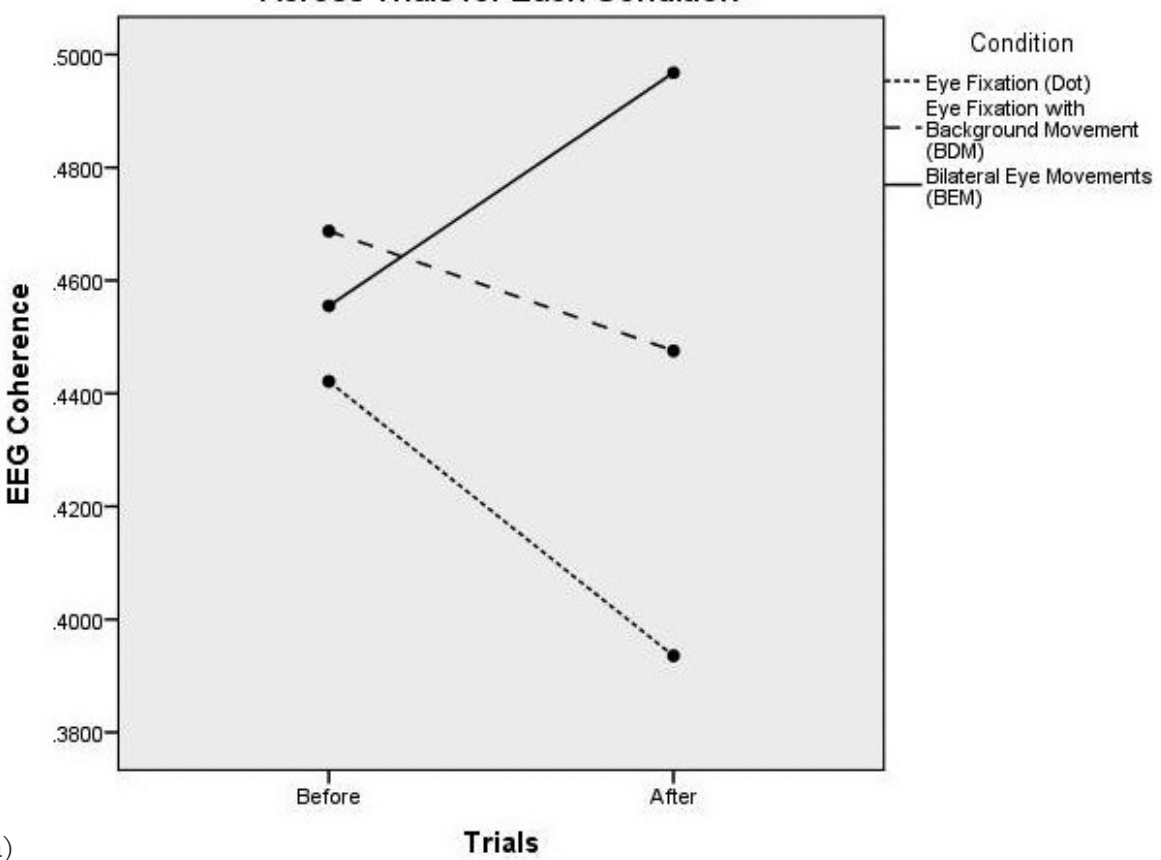

Right Frontal Beta Intrahemispheric Coherence Changes Across Trials for Each Condition

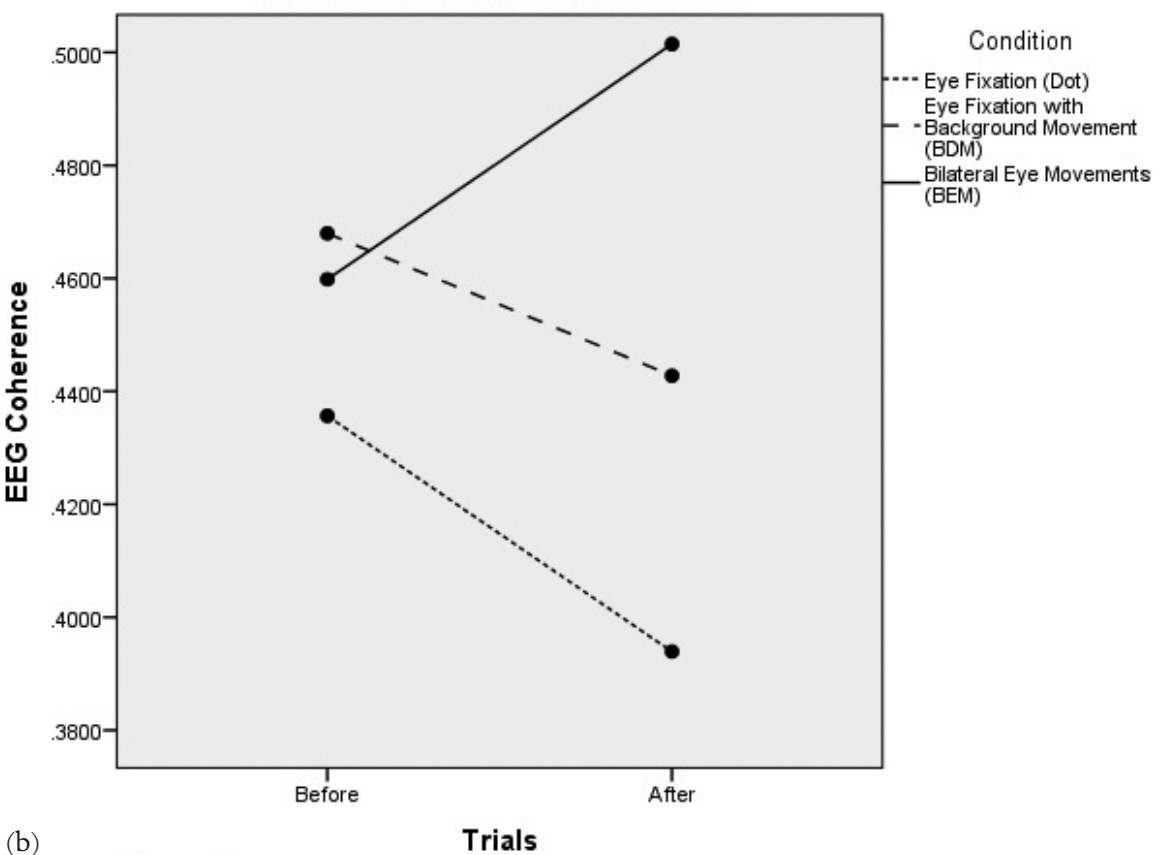

FIGURE 4. (a) Changements de la cohérence bêta intrahémisphérique frontale gauche lors des différents essais pour chaque condition. (La cohérence Point a diminué significativement ; la cohérence MOB a montré une tendance non significative à augmenter.) (b) Changements de la cohérence bêta intrahémisphérique frontale droite lors des différents essais pour chaque condition. (La cohérence MOB a augmenté significativement ; la cohérence Point a diminué significativement.)

Note: Left Frontal Beta Intrahemispheric Coherence Changes Across Trials for Each Condition = changements de la cohérence bêta intrahémisphérique frontale gauche lors des différents essais pour chaque condition; Right Frontal Beta Intrahemispheric Coherence Changes Across Trials for Each Condition = changements de la cohérence bêta intrahémisphérique frontale droite lors des différents essais pour chaque condition; Condition = condition ; Eye Fixation (Dot) = Yeux fixes (Point); Eye Fixation with Backgound Movement $(\mathrm{BDM})=$ yeux fixes avec un mouvement en arrière-plan (MAP) ; Bilateral Eye Movements $(\mathrm{BEM})=$ mouvements oculaires bilatéraux $(\mathrm{MOB}) ; \mathrm{EEG}$ Coherence $=$ cohérence EEG ; Trials $=$ essais $;$ Before $=$ avant $;$ After $=$ après. 


\section{Right Frontal Gamma Intrahemispheric Coherence Changes Across Trials for Each Condition}

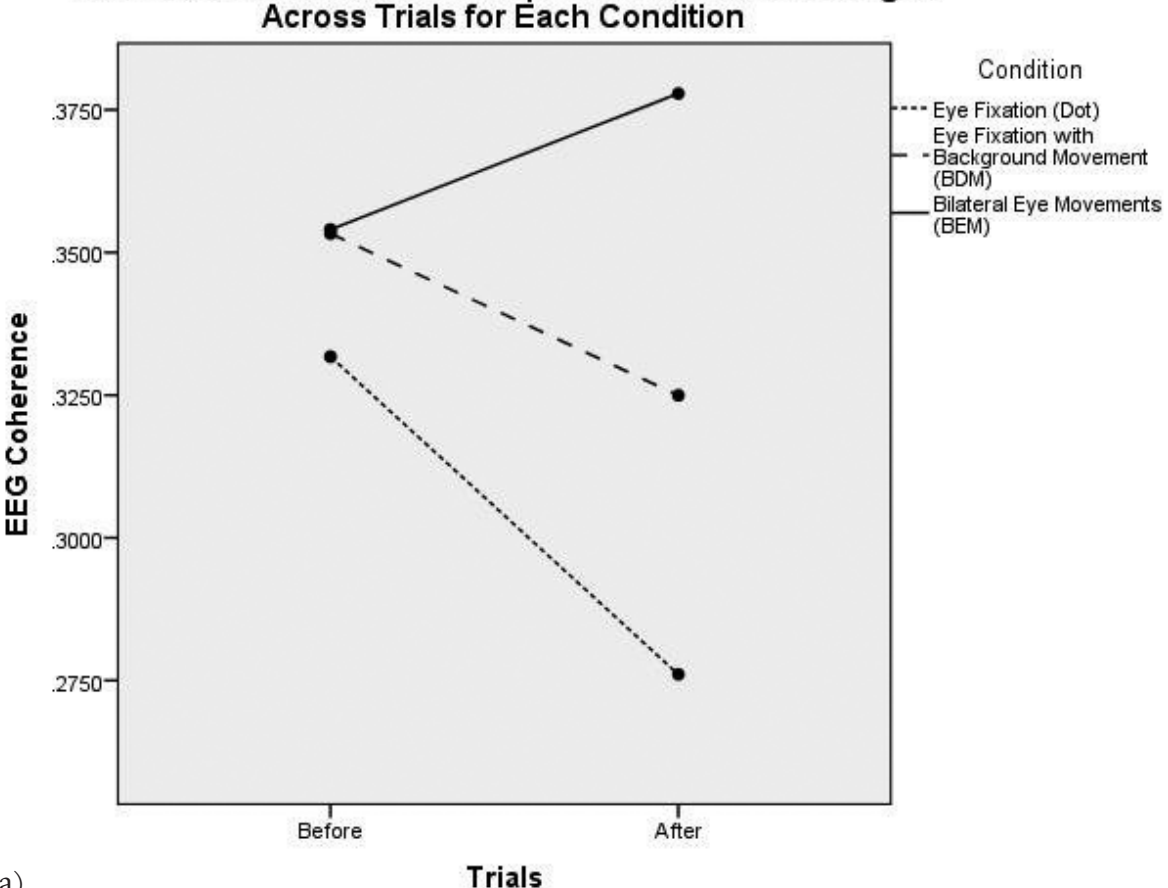

(a)

Trials

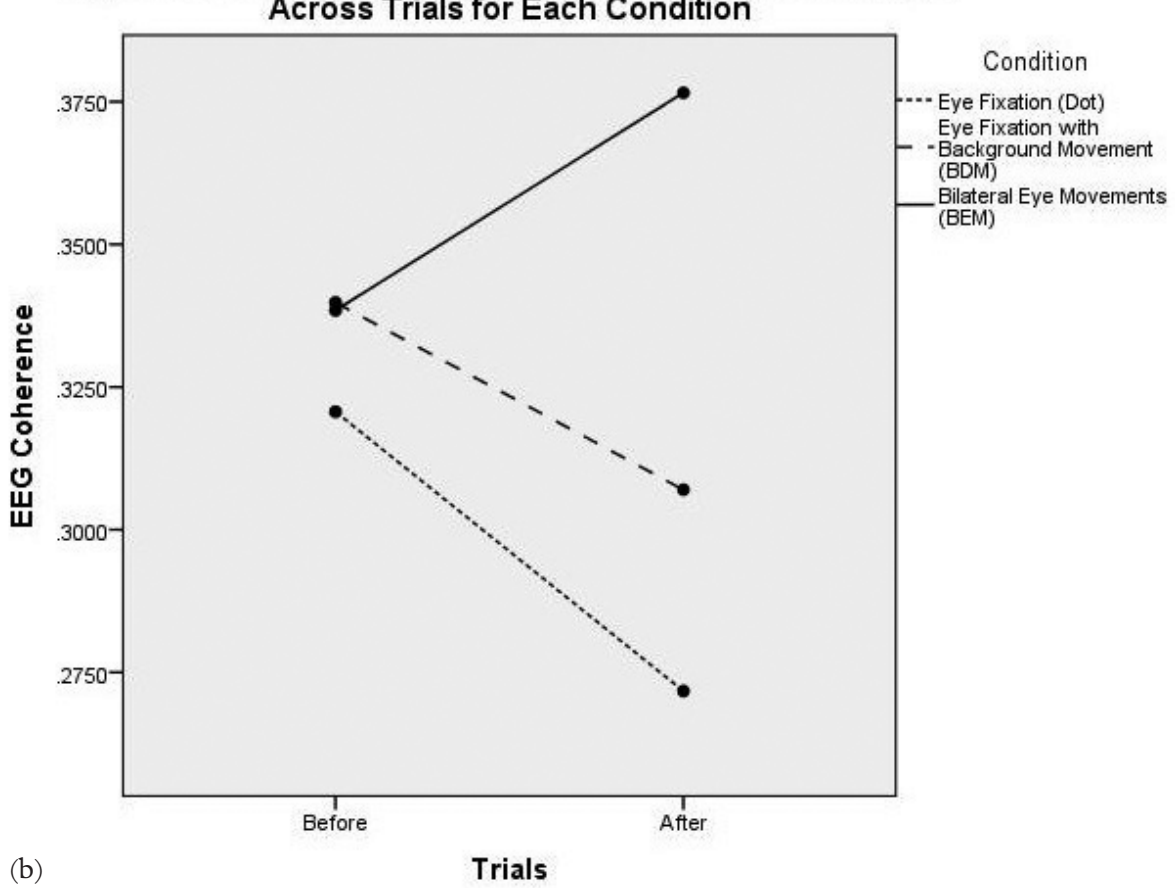

(b)

Trials

FIGURE 5. (a) Changements de la cohérence gamma intrahémisphérique frontale gauche lors des différents essais pour chaque condition. (La cohérence Point a diminué significativement ; la cohérence MOB a montré une tendance non significative à augmenter.) (b) Changements de la cohérence gamma intrahémisphérique frontale droite lors des différents essais pour chaque condition. (La cohérence Point a diminué significativement ; la cohérence MOB a montré une tendance non significative à l'augmentation.)

Note: Left Frontal Gamma Intrahemispheric Coherence Changes Across Trials for Each Condition = changements de la cohérence gamma intrahémisphérique frontale gauche lors des différents essais pour chaque condition ; Right Frontal Gamma Intrahemispheric Coherence Changes Across Trials for Each Condition $=$ changements de la cohérence gamma intrahémisphérique frontale droite lors des différents essais pour chaque condition; Condition = condition ; Eye Fixation (Dot) $=$ Yeux fixes $($ Point $)$; Eye Fixation with Backgound Movement $(\mathrm{BDM})=$ yeux fixes avec un mouvement en arrière-plan (MAP) ; Bilateral Eye Movements (BEM) = mouvements oculaires bilatéraux (MOB); EEG Coherence $=$ cohérence EEG $;$ Trials $=$ essais $;$ Before $=$ avant $;$ After $=$ après . 


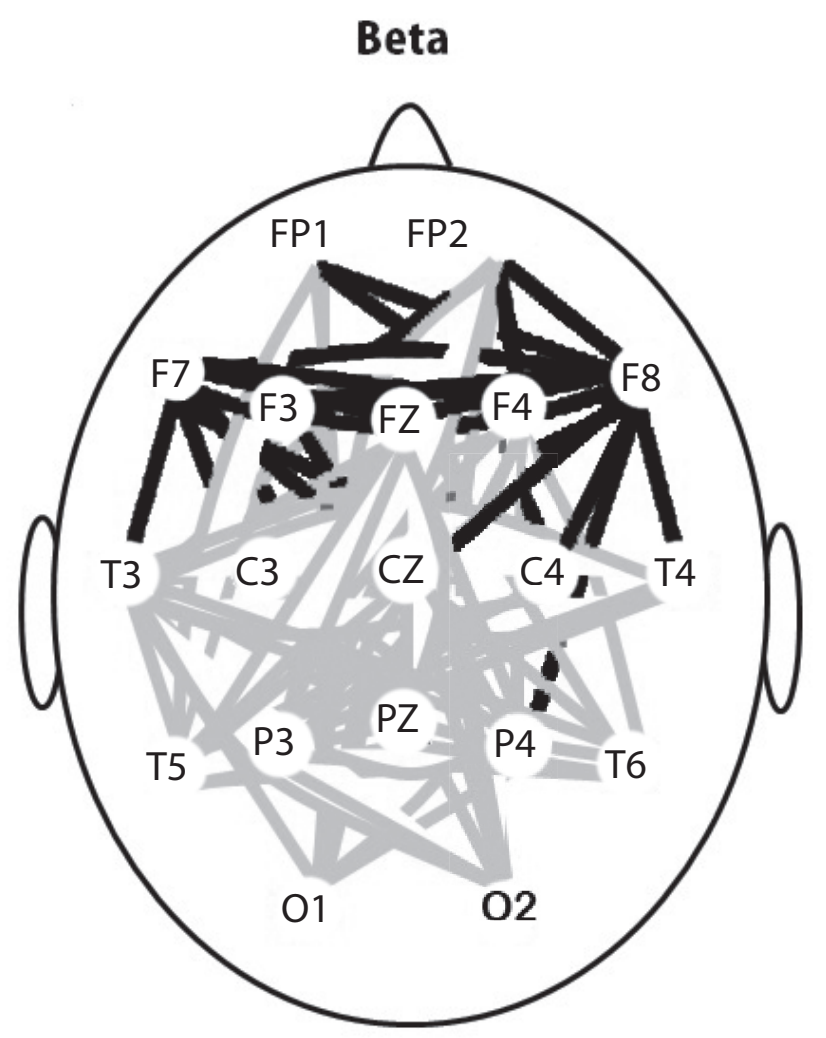

FIGURE 6. Carte des cohérences pour les changements de cohérence corticale bêta entre le prétraitement et le posttraitement pour la condition de mouvements oculaires bilatéraux. (Les lignes noires entre les pairs d'électrodes représentent des augmentations de la cohérence EEG et les lignes grises représentent des diminutions de la cohérence. Notez les augmentations plus prononcées de l'hémisphère droit frontal après les MOB.)

Ces résultats suggèrent que les effets des associations libres avec un souvenir désagréable initial suivant des MOB en contemplant ce souvenir, comme cela se produit dans la phase 4 (la désensibilisation de l'EMDR), sont d'augmenter principalement la cohérence EEG thêta et bêta frontale droite. Et, dans cette étude, ce qui coïncidait avec ces effets de cohérence mais qui se produisait également dans les conditions de contrôle, était des diminutions significatives de la vivacité de l'imagerie et de la valence émotionnelle du souvenir désagréable initial.

\section{Cohérence interhémisphérique}

Cette étude n'a pas obtenu l'augmentation prédite de la cohérence interhémisphérique au cours d'une période d'association libre immédiatement après la stimulation bilatérale au cours du rappel des détails d'un souvenir épisodique désagréable. Ces résultats sont cohérents avec ceux de l'étude de Keller et coll. (2014) ainsi qu'avec les investigations portant sur la cohérence de Propper et coll. (2007) et de Samara et coll. (2011). Néanmoins, la présente investigation a observé une augmentation légèrement non significative de la cohérence interhémisphérique bêta frontale après la participation à la condition MOB. Ce résultat pourrait laisser penser à une tendance au renforcement de la cohérence interhémisphérique pendant la contemplation et les associations libres à un souvenir désagréable mais non traumatique. En effet, des effets de renforcement de la cohérence EEG interhémisphérique peuvent présenter une amplitude faible (c.-à-d. la taille d'effet) et être à peine détectables, requérant une grandeur d'effet importante pour atteindre la signification statistique, c'est-à-dire un événement traumatique marquant et/ou un grand échantillon de participants pour réduire la variabilité.

Les raisons de la cohérence réduite dans la condition Point pour les fréquences bêta et gamma ne sont pas claires et seront envisagées plus loin dans cette discussion. Il est toutefois intéressant de noter que ce changement ne s'est pas produit dans la condition MOB. Nous ne pouvons que spéculer à ce stade-ci que ces observations d'augmentations non significatives de la cohérence interhémisphérique bêta frontale auraient pu être plus prononcées avec un événement traumatique personnellement significatif, plus caractéristique de la psychothérapie EMDR.

\section{Cohérence intrahémisphérique}

Cohérence intrahémisphérique bêta et gamma frontale. Les effets différentiels de la condition sur la cohérence intrahémisphérique étaient considérablement plus remarquables. En se référant aux Figures 4 et 5 , il apparaît clairement une tendance unique mais assez constante des cohérences bêta et gamma frontales, tant pour l'hémisphère droit que pour l'hémisphère gauche. Même si les effets de la condition MOB ne sont statistiquement significatifs que pour une augmentation de la bêta frontale droite, la cohérence MOB augmente de manière descriptive dans les régions frontales gauche et droite pour les fréquences EEG à la fois bêta et gamma. Ces augmentations deviennent encore plus saillantes en la présence de diminutions statistiquement significatives de la cohérence pour la condition Point contrôle pour les deux hémisphères et les deux fréquences. Et même la condition MAP de contrôle montre des diminutions cohérentes mais non significatives pour chacune de ces variables.

Nous demeurons assez perplexes quant à la raison précise qui expliquerait pourquoi les deux conditions de contrôle seraient associées à une cohérence réduite pour ces fréquences et ces régions corticales. 
Cependant, étant donné que la cohérence reflète l’interconnectivité et l'intercommunication neuronales, ces diminutions reflètent l'inverse, c'est-à-dire une déconnexion entre paires de sites d'enregistrement par la fixation d'un point, avec ou sans mouvements bilatéraux en arrière-plan. Une interprétation de cette non connectivité pourrait correspondre à des associations plus aléatoires avec des informations moins en lien avec le souvenir cible. Une autre façon de décrire ce phénomène pourrait être un esprit qui vagabonde de manière non pertinente.

D’un autre côté, la cohérence accrue observée pour la condition MOB suggère que les liens associatifs étaient davantage centrés sur des informations plus pertinentes par rapport à l'événement cible. Il est intéressant de noter que les effets significatifs des MOB se produisaient relativement à des diminutions marquées et cohérentes de la cohérence corticale après le fait d'être simplement assis en fixant un point, ce qui laisse penser que les stimulations $\mathrm{MOB}$ renforce le traitement de souvenirs désagréables personnellement significatifs en comparaison de ce qui se produirait plus naturellement dans une condition neutre. De plus, le fait que ces effets différentiels ne se produisaient que pour les bandes bêta et gamma indique que les régions corticales identifiées étaient activées par des fréquences EEG associées à un traitement de l'information d'ordre supérieur, à un état alerte et à une attention ciblée (Bergmann, 2008).

Localisations corticales de la cohérence EEG renforcée. Ces résultats indiquent une cohérence accrue dans des régions limitées du cortex pendant les associations libres suivant des MOB en se rappelant un souvenir désagréable, de manière similaire aux résultats obtenus par Keller et coll. (2014). En effet, comme pour Keller et coll., les aires corticales activées par les stimulations MOB dans cette étude étaient des régions impliquées dans des tâches exécutives de traitement de l'information intervenant dans la prise de décisions, la planification, les conduites prosociales, la différenciation entre expériences positives et négatives, et l'expression de la personnalité. De plus, la neuroimagerie de la cohérence associée à ces résultats dépeint une connectivité corticale accrue parmi sept sites d'électrodes frontaux pour la fréquence bêta, avec des extensions dans plusieurs sites centraux et temporaux, et un site pariétal, plus particulièrement dans l'hémisphère droit.

Cette implication plus importante de l'hémisphère droit au cours du traitement de souvenirs désagréables est aussi cohérente avec les résultats obtenus par Keller et coll. (2014) sur des souvenirs positifs.
Ces chercheurs ont montré une cohérence bêta intrahémisphérique frontale gauche et droite et une activation par neuroimagerie LORETA bêta basse préfrontale droite au cours d'associations libres suivant des MOB pendant le rappel d'un souvenir positif. Ces résultats portant sur une activation prédominante des aires préfrontales droites pourraient représenter une implication accrue des composants affectifs associés au retraitement de l'événement cible.

En effet, notre court résumé des études de neuroimagerie corticale sur l'EMDR, figurant plus haut, indiquent une activation des régions frontomédiales et frontolatérales et une désactivation des régions pariétales et occipitales plus postérieures après la psychothérapie EMDR (voir la Figure 6 pour comparaison). Et même si des comparaisons directes entre notre étude et l'étude EEG longitudinale sur l'EMDR de Pagani et coll. (2012) sont difficiles en raison des différences de mesures, d'équipement, de tâches et de procédures, ces chercheurs ont trouvé une activation corticale significativement plus élevée dans le gyrus fusiforme droit, près de l'électrode T4 (voir Figure 6) au court de l'écoute de scripts traumatiques, après une psychothérapie EMDR. Nos deux études de cohérence associées aux études de neuroimagerie corticale analysées auparavant sont cohérentes avec une activation accrue, par les stimulations bilatérales et les composants de retraitement subséquents de l'EMDR, de régions corticales frontales impliques dans le retraitement cognitif et sensoriel de régions limbiques hyperréactives et isolées, activées par des expériences traumatiques.

Cohérence thêta intrahémisphérique frontale. Nous avons également observé une augmentation imprévue de la cohérence thêta intrahémisphérique frontale droite pour la condition MOB. Les ondes thêta sont intéressantes dans les études d'EEG fonctionnelle car elles sont associées à l'implication imaginaire, à la créativité, à la résolution de problèmes, au traitement perceptuel, à la performance attentive dans les tâches cognitives et aux états dissociatifs (Stevens et coll., 2004) ainsi qu'au traitement de souvenirs épisodiques (Klimesch, 1999). Par conséquent, l'observation d'une cohérence accrue pour cette forme d'onde lente $(4-8 \mathrm{~Hz})$ dans les régions frontales droites suivant la condition MOB reflète très probablement un traitement imaginatif et perceptuel autoréférencé des composants affectifs du souvenir désagréable ciblé.

\section{Condition yeux fixes avec mouvements en arrière-plan}

Notre étude a inclus une condition de contrôle assez unique, l'expérience MAP. Nous avons inclus cette 
condition pour tester si les mouvements oculaires étaient nécessaires pour affecter les changements de cohérence corticale ou si une stimulation bilatérale du champ visuel suffisait. L'observation du Tableau 1 et des Figures 4 et 5 indiquent que la condition MAP tendait à ressembler plutôt à la condition Point seul, suggérant que notre stimulation bilatérale en arrière-plan ne suffisait pas à déclencher les effets observés d'augmentation de la cohérence dans la condition MOB. Il apparait que l'acte physique des saccades bilatérales peut être requis pour favoriser une connectivité neurale accrue. Bien sûr, de futures recherches seront nécessaires pour examiner si d'autres formes de stimulations bilatérales, auditives ou tactiles, auront des effets EEG similaires (Nieuwenhuis et coll., 2013).

\section{Changements de la vivacité de l'image et de la} valence émotionnelle associées au souvenir

Nous avons observé des diminutions progressives de la vivacité de l'image désagréable et de la valence émotionnelle négative dans les trois conditions de notre étude. Ces résultats pour les MOB sont cohérents avec des études antérieures sur les effets plus immédiats des saccades bilatérales sur la vivacité principalement sémantique de souvenirs rapportés par de nombreux autres chercheurs (voir Van den Hout, Eidhof, Verboom, Littel \& Engelhard, 2013 ; Van den Hout \& Engelhard, 2012 pour des résumés de cette littérature). Néanmoins la détérioration de la vivacité des souvenirs dans notre étude ne reflétait pas les différences significatives entre les conditions MOB et de contrôle observées dans la plupart des études. Il se peut que les effets de notre étude étaient modérés par l'expérience subséquente d'associations libres qui n'était pas employée dans les autres études. De plus, même si notre étude demandait aux participantes d'évaluer la vivacité et la valence émotionnelle du souvenir initial, ces évaluations étaient effectuées après la phase d'association libre du processus dans les trois conditions et les participantes ont pu incorporer le matériel associé au souvenir initial pendant leurs évaluations.

La séquence répétée de dégradation du souvenir initial rappelé par le processus de stimulation bilatérale et par l'obtention de matériel associé pendant les associations libres a été expliquée par Maxfield, Melnyk et Hayman (2008). Ce processus offre une explication de la diminution que nous avons observée au niveau de la vivacité de l'image et de la valence émotionnelle pour la condition MOB. Par contre, cet effet obtenu pour les autres conditions, pour lesquelles on ne s'attendrait pas à une saturation similaire du calepin visuospatial de la mémoire de travail, demeure difficile à expliquer, à moins que le processus d'association libre sollicite de manière similaire la mémoire de travail.

Comparaisons avec l'étude de Keller et coll. (2014). Les résultats de notre étude sur des souvenirs désagréables contredisent notre étude antérieure sur des souvenirs agréables, qui a montré une force accrue du souvenir et une plus grande vivacité dans les trois conditions (Keller et coll., 2014). Samara et coll. (2011) et Nieuwenhuis et coll. (2013) ont également montré un rappel amélioré de mots à valence négative avec la stimulation bilatérale mais pas dans les conditions de contrôle. Et comme nous l'avons déjà noté, plusieurs études antérieures ont rapporté des augmentations du rappel de souvenirs à la fois sémantiques et épisodiques avec la présentation séquentielle de stimulation bilatérale et de tests mnésiques mais une vivacité et une valence émotionnelle réduites après une stimulation bilatérale et un test mnésique concomitants. Ces études peuvent difficilement être comparées à celle de Keller et coll. (2014) et à celle-ci car aucune de ces investigations antérieures n'apportait de phase de traitement ou d'association libre après la stimulation bilatérale. L'exception, comme nous l'avons déjà noté, était l'étude de Lee et Drummond (2008) qui a montré une diminution de la vivacité du souvenir immédiatement après la désensibilisation, seulement dans les conditions de mouvements oculaires et de distanciation.

Nous avons noté dans Keller et coll. (2014) que les effets obtenus pouvaient résulter du fait que notre investigation mesurait la force et la vivacité après le traitement du souvenir positif cible plutôt qu'immédiatement après la stimulation bilatérale. Comme cette étude-là a observé une vivacité accrue quelle que soit la condition et que des études antérieures avaient obtenu une diminution différentielle propre aux MOB, nous avons suggéré que nos résultats reflétaient plutôt un effet du traitement qu'un effet des SBL. Nous proposons que la même chose s'applique dans cette étude-ci : la différence pourrait être liée à la nature du souvenir rappelé, c'est-à-dire à sa qualité positive ou négative, comme nous l'expliciterons ci-après.

\section{Formulation proposée de la dynamique de l'EMDR}

Ces spéculations ne contredisent pas notre formulation de la dynamique de l'EMDR proposée par Keller et coll. (2014), Maxfield et coll. (2008) et d'autres. Nous avions suggéré que le composant de la stimulation bilatérale opère à travers la déflation d'un événement mnésique résultant d'une saturation de la mémoire de travail par la tâche consistant simultanément à prêter attention à un souvenir cible et à suivre une forme de 
stimulation bilatérale. Ceci serait alors suivi du retraitement subséquent de la trace mnésique maintenant affaiblie par le biais de sa connexion à des liens associatifs éloignés mais apparentés à travers des voies neurales activées par le processus de stimulation bilatérale. Ce retraitement renforcerait un souvenir initialement positif ou contribuerait à la création d'un recadrage constructif pour un souvenir négatif encore affaibli. Sans aucun doute, ces spéculations constituent un appel à des analyses séquentielles des composants de nature cognitive et électrophysiologique.

Modèle de cohérence corticale en deux étapes. Nous avons commencé cet article par une très brève présentation des nombreuses théories avancées pour expliquer l'effet des mouvements oculaires sur l'efficacité de l'EMDR. Nous aimerions ici proposer, avec prudence, une intégration de ces nombreuses théories dans un modèle de cohérence corticale en deux étapes pour l'EMDR. Il est certain qu'à l'heure actuelle, ce modèle est une proposition partiellement fondée sur les résultats de seulement deux études et qu'il manque de validation empirique. Si des études de cohérence ultérieures de la part d'autres laboratoires confirment nos résultats d'augmentation de la cohérence corticale pendant le traitement de souvenirs traumatiques suivant une stimulation bilatérale, alors il apparaîtrait qu'en effet, la stimulation bilatérale, du moins en partie, favorise le retraitement des souvenirs en augmentant l'interconnectivité neurale.

Cette connectivité neurale renforcée permet à des liens associatifs plus nombreux à se former entre des souvenirs traumatiques affaiblis par imagination/déflation et des recadrages plus positifs et constructifs au cours de l'association libre et d'autres expériences de retraitement. Un tel modèle de cohérence corticale en deux étapes inclut et intègre aisément les modèles de conditionnement de Dyck (1993), Denny (1995), Armstrong et Vaughan (1996) et MacCulloch et Feldman (1996), les modèles imagination déflation mémoire de travail calepin visuospatial/administrateur central d'Andrade et coll. (1997) et de Gunter et Bodner (2008), ainsi que les modèles de connectivité physiologique de Bergmann (1998, 2000), Servan-Schreiber (2000), Stickgold (2002), Corrigan (2002) et Rasolkhani-Kalhorn et Harper (2000).

De plus, on peut concevoir que certains de ces modèles s'appliquent mieux à une étape donnée du processus EMDR, avec le modèle de la mémoire de travail représentant le mieux la désensibilisation de la trace mnésique traumatique et les modèles de conditionnement et de connectivité décrivant mieux le retraitement, le recadrage et l'intégration de liens associatifs nouvellement formés dans la conscience.
Comme mentionné dans notre article antérieur (Keller et coll., 2014), un tels modèle intégré en deux étapes pour l'EMDR reflète la sélection très intuitive des termes de désensibilisation et de retraitement par le Dr Shapiro voici près de trente ans.

\section{Limitations de la présente étude}

Cette investigation comporte plusieurs limitations qui restreignent les généralisabilité et la précision des résultats. La limitation la plus importante provient de la taille de l'échantillon. Les études EEG sont très exigeantes en termes de ressources, nécessitant non seulement des procédures très sophistiquées et précises d'application des électrodes et de configuration de l'équipement, mais aussi des protocoles de traitement des données très chronophages. Par conséquent, elles tendent à impliquer un nombre de participants plus réduit que les études psychologiques typiques. Même si notre investigation était de taille légèrement supérieure à celle des études EEG résumées auparavant, nos chiffres compromettaient la puissance et résultaient en un nombre considérable d'effets apparents qui n'étaient pas significatifs mais qui reflétaient les tendances des résultats de cohérence soutenant les hypothèses. Un échantillon de taille supérieure aurait pu permettre une moindre variabilité de l'échantillon, des effets spécifiques plus significatifs et un moindre risque d'erreur de type 1 , mais aussi l'émergence de fréquences et de localisations corticales ayant des implications importantes pour les effets MOB.

De plus, étant donné nos résultats de différences de groupes au niveau de la cohérence EEG intrahémisphérique en l'absence de telles différences sur le plan de la vivacité de l'image et de la valence émotionnelle des souvenirs désagréables, il nous est difficile de commenter la nature de ces relations dans notre étude. Clairement, si des liens associatifs se forment pendant les MOB plus que dans nos conditions de contrôle, nous aurions dû observer des effets différentiels de la condition sur la vivacité et la valence des souvenirs. Les différences subtiles des tendances, en l'absence d'effets statistiques, ont pu être fonction de la taille de l'échantillon et/ou d'une petite taille d'effet de notre brève intervention sur des souvenirs non traumatiques. Les recommandations figurant ci-après tenteront d'aborder ce décalage.

Bien que la décision de ne pas ajuster le taux d'erreur famille pour les analyses EEG ait pu faire courir le risque d'une certaine inflation de l'erreur de type 1 pour chaque analyse, cet effet est plus ou moins compensé par la réduction des degrés de liberté dans l'analyse covariée. Nous pensons également que les données indiquant des tendances stables au niveau 
des résultats d'études plus réduites ont une valeur indéniable, même en l'absence d'une signification statistique. Comme dans toute recherche investigatrice, les résultats obtenus seront provisoires jusqu'à leur confirmation par une réplication indépendante. Nous espérons que nos résultats stimuleront de futures recherches qui testeront nos hypothèses.

Nous avons limité notre échantillon à des femmes droitières pour rendre les effets potentiels de la latéralité de référence liée au sexe aussi homogènes que possible. Ce biais de sélection limite bien sûr encore plus la généralisabilité. Notre étude a aussi utilisé 19 sites d'électrodes corticaux. Les montages d'électrodes EEG varient en termes de densité et les études EEG humaines ont été réalisées avec des configurations possédant jusqu'à 256 électrodes. Nous aurions pu améliorer la résolution de nos résultats EEG, particulièrement en ce qui concerne la localisation corticale, si nous avions employé une configuration d'électrodes plus dense. Bien sûr, des montages plus denses impliquent un temps d'application plus long, un risque accru liés aux dérivations et aux pannes des électrodes, ainsi qu'une attention plus grande aux différences d'impédance, facteurs qui contribuent tous à augmenter le coût de la réponse de la portion EEG de l'étude.

\section{Recommandations pour les recherches futures}

À la suite des commentaires dans le texte qui précède, nous recommanderions de reproduire cette étude dans un laboratoire indépendant avec une taille d'échantillon considérablement plus importante, avec des hommes et des femmes, en prenant soin de déterminer leur latéralisation avec précision, et avec une configuration d'électrodes EEG un peu plus dense, par exemple, 36-40 électrodes. Et comme l'objectif de ce type de recherche consiste à mieux comprendre les mécanismes neurologiques des effets de l'EMDR dans le traitement du trauma, particulièrement en ce qui concerne le composant de la stimulation bilatérale, des études doivent être menées utilisant des populations cliniques ayant vécu des expériences traumatiques plus sévères. Des études EEG cliniques longitudinales sur la psychothérapie EMDR, ressemblant davantage aux ambitieux projets de Pagani et coll. (Pagani et coll., 2012 ; Pagani et coll., 2013) sont fortement recommandées.

De plus, étant donné les résultats de Nieuwenhuis et coll. (2013) concernant le rappel amélioré de mots à valence négative suivant une stimulation bilatérale oculaire et tactile, mais pas auditive, il serait intéressant de reproduire notre étude de manière constructive pour comparer les effets de ces trois types de stimulation bilatérale sur la cohérence inter- et intrahémisphérique. Au vu des effets de renforcement de la cohérence intrahémisphérique par la stimulation bilatérale oculaire dans notre étude et selon les résultats de Nieuwenhuis et coll. (2013), nous prédirions un résultat similaire avec la stimulation bilatérale tactile mais pas avec la stimulation bilatérale auditive.

Nous sommes certainement d'accord avec l'importance de la recherche fondamentale sur les composants de la psychothérapie EMDR, comme les études sur la stimulation bilatérale de Christman et de ses collègues, de Van den Hout et de ses associés, de Gunter et Bodner, de Maxfield et de ses collègues, de Samara et de ses associés, et d'autres mentionnées auparavant. En effet, ces études ont joué un rôle essentiel permettant une meilleure compréhension des contributions de ces composants fondamentaux. Et même, comme mentionné auparavant, il serait intéressant d'examiner les différences de traitement entre souvenirs positifs et négatifs dans une seule étude, tant sur le plan électrophysiologique que sur le plan comportemental.

Toutefois, nous avons le sentiment que la synergie thérapeutique de plusieurs aspects du travail clinique peut parfois être perdue dans l'examen des composants en dehors du contexte du processus psychothérapeutique. Nous avons tenté d'examiner un ensemble de tels aspects synergiques de la phase 4 (la désensibilisation du protocole de la psychothérapie EMDR), comme l'ont fait Lee et Drummond (2008), c'est-à-dire les MOB, l'attention concomitante à une expérience désagréable gardée en mémoire de travail et le processus subséquent de traitement par association libre de cet événement désagréable. Bien que la comparaison directe de nos résultats à un examen plus microscopique de chaque composant sorti de son contexte puisse présenter un intérêt limité, nous avons le sentiment que la pertinence pour le processus psychothérapeutique de l'EMDR pourrait être accrue par une telle analyse et pourrait augmenter la validité externe de ces résultats. Par conséquent, nous encourageons de telles études futures portant sur le processus clinique de l'EMDR, mais peut-être avec des mesures EEG et de la vivacité du souvenir et de la valence à de multiples points du processus, particulièrement avant et après le traitement par association libre.

\section{Application potentielle pour le traitement clinique des traumatismes crâniens}

Enfin, nous aimerions proposer une spéculation supplémentaire, très hypothétique, à partir des résultats de nos deux études sur la cohérence. À notre connaissance, il n'y a pas eu d'investigation publiée sur l'application de l'EMDR ou de la stimulation 
bilatérale au traitement des symptômes des traumatismes crâniens (TC).

Les analyses de Kennedy et coll. (2007) et de Tanev, Pentel, Kredlow et Charney (2014) n'ont pas non plus trouvé d'examens publiés de l'EMDR ou d'autres traitements TCC pour les TC. L'EMDR a bien sûr été appliqué au traitement de l'ESPT, et l'ESPT et le TC sont très souvent concomitants, particulièrement chez les vétérans de guerre (Kennedy et coll., 2007 ; Tanev et coll., 2014 ; Vasterling, Verfaellie \& Sullivan, 2009). Le groupe de travail cognitif du Collège américain de médecine de réhabilitation a analysé la littérature sur le traitement des TC entre 2003 et 2008 ; il a conclu que la réhabilitation cognitive (entraînement de l'attention et stratégies métacognitives avec une validité externe) est plus efficace que la réhabilitation conventionnelle et constitue le meilleur traitement des séquelles des TC (Cicerone et coll., 2011).

Une raison pour laquelle l'EMDR et les autres stratégies TCC ne sont pas appliqués au traitement des symptômes de TC pourrait être que les capacités cognitives détériorées de la plupart de personnes atteintes d'un TC sembleraient constituer un facteur restrictif dans l'application de stratégies de traitement nécessitant un fonctionnement cognitif intact (Tanev et coll., 2014). Cependant, si des réplications constructives de nos études indiquant une connectivité neuronale accrue après une stimulation bilatérale soutiennent le modèle de cohérence corticale, alors la stimulation bilatérale apportée pendant la réalisation de tâches cognitives compromises par le TC (mémoire, fonctions motrices, traitement exécutif, etc.) pourrait peut-être accélérer la récupération de ces fonctions. Il est certain que des études des effets de l'EMDR formel sur les résultats de tests neuropsychologiques ainsi que de la cohérence EEG chez des patients atteints d'un ESPT et d'un TC comorbides pourraient suggérer l'ajout d'un composant de traitement par stimulation bilatérale aux programmes de réhabilitation cognitive pour le traitement d'un TC primaire.

\section{NOTES}

1. Un plan intrasujets typiquement plus puissant, comme celui utilisé par Samara et coll. (2011) pour le rappel de mots neutres et émotionnels, n’a pas été employé dans cette étude en raison de préoccupations concernant les effets de report des conditions de traitement sur la vivacité et la valence émotionnelle ainsi que sur les effets électrocorticaux, en rapport avec les souvenirs épisodiques désagréables et personnellement significatifs utilisés dans cette étude. En effet, Keller et coll. (2014) ont observé des augmentations de la vivacité et de la valence émotionnelle de souvenirs épisodiques positifs après chaque présentation du stimulus pour les trois conditions de leur étude.

2. Pour examiner plus avant la possible contribution d'artéfacts résiduels des muscles oculaires aux effets EEG du pôle frontal, ces analyses ont été répétées avec l'omission des électrodes Fp1 et Fp1 dans le groupe frontal. La même répartition de tendances et d'effets significatifs était obtenue dans cette nouvelle analyse.

\section{Bibliographie}

Aitken, R. C. (1969). Measurement of feelings using visual analogue scales. Journal of the Royal Society of Medicine, 62(10), 989-993.

Andrade, J., Kavanagh, D., \& Baddeley, A. (1997). Eye movements and visual imagery: A working memory approach to the treatment of post-traumatic stress disorder. British Journal of Clinical Psychology, 36, 209-223.

Armstrong, M. S., \& Vaughan, K. (1996). An orienting response model of eye movement desensitization. Journal of Behavior Therapy and Experimental Psychiatry, 27, 21-32.

Barenne, D. D., \& Gibbs, F. A. (1942). Variations in the electroencephalogram during the menstrual cycle. American Journal of Obstetrics and Gynecology, 44, 687-690.

Bergmann, U. (1998). Speculations on the neurobiology of EMDR. Traumatology, 4, 4-16.

Bergmann, U. (2000). Further thoughts on the neurobiology of EMDR: The role of the cerebellum in accelerated information processing. Traumatology, 6(3), 175-200.

Bergmann, U. (2008). The neurobiology of EMDR: Exploring the thalamus and neural integration. Journal of EMDR Practice and Research, 2(4), 300-314.

Bremner, J. D. (2007). Functional neuroimaging in posttraumatic stress disorder. Expert Review of Neurotherapeutics, 7, 393-405.

Brown, R. E. (1994). An introduction to neuroendocrinology. Cambridge, MA: Cambridge University Press.

Carlson, E. B., \& Putnam, F. W. (1993). An update on the dissociative experiences scale. Dissociation, 1, 16-27.

Christman, S. D., Propper, R. E., \& Dion, A. (2004). Increased interhemispheric interaction is associated with decreased false memories in a verbal converging semantic associates paradigm. Brain and Cognition, 56, 313-319.

Cicerone, K. D., Langenbahn, D. M., Braden, C., Malec, J. F., Kalmar, K., Fraas, M., . . Ashman, T. (2011). Evidencebased cognitive rehabilitation: Updated review of the literature from 2003 through 2008. Archives of Physical Medicine and Rehabilitation, 92, 519-530.

Corrigan, F. (2002). Mindfulness, dissociation, EMDR, and the anterior cingulate cortex. Contemporary Hypnosis, 19(1), 8-17.

Denny, N. (1995). An orienting reflex/external inhibition model of EMDR and thought field therapy. Traumatology, 1(1), 1-6.

Dyck, M.J. (1993). A proposal for a conditioning model of eye movement desensitization treatment for post- traumatic 
stress disorder. Journal of Behavior Therapy and Experimental Psychiatry, 24(3), 201-210.

ElectroCapSystem[appareil](1983).Eaton,OH:Electro-Cap International. Téléchargé depuis http://www.electrocap.com

EureKa! [logiciel] (2000). Mesa, AZ: Nova Tech EEG. Téléchargé depuis http: / www.novatecheeg.com

EyeScan 2000S Light Bar [appareil] (1994). Wheat Ridge, CO: NeuroTek. Téléchargé depuis http://www .neurotekcorp.com

Francati, V., Vermetten, E., \& Bremner, J. D. (2007). Functional neuroimaging studies in post-traumatic stress disorder: Review of current methods and findings. Depression and Anxiety, 24, 202-218.

Frederick, J. A. (2001). Open source method of graphical QEEG analysis using PERL and Visual Basic. Journal of Neurotherapy, 4(4), 39-47.

Gunter, R. W., \& Bodner, G. E. (2008). How eye movements affect unpleasant memories: Support for a Working-memory account. Behaviour Research and Therapy, 46, 913-931.

Horowitz, M., Wilner, N., \& Alvarez, W. (1979). Impact of event scale: A measure of subjective distress. Psychometric Medicine, 41, 209-218.

Kaplan, B. J., Whitsett, S. F., \& Robinson, J. W. (1990). Menstrual cycle phase is a potential confound in psychophysiology research. Psychophysiology, 27, 445-450.

Keller, B., Stevens, L., Lui, C., Murray, J., \& Yaggie, M. (2014). The effects of bilateral eye movements on EEG coherence when recalling a pleasant memory. Journal of EMDR Practice and Research, 8(3), 113-128.

Kennedy, J. E., Jaffee, M. S., Leskin, G. A., Strokes, J. W., Leal, F. O., \& Fitzpatrick, P. J. (2007). Posttraumatic stress disorder and posttraumatic stress disorder-like symptoms and mild traumatic brain injury. Journal of Rehabilitation Research and Development, 44(7), 1-30.

Klimesch, W. (1999). EEG alpha and theta oscillations reflect cognitive and memory performance: A review and analysis. Brain Research Reviews, 29, 169-195.

Knott, V. J., LaBelle, A., Jones, B., \& Mahoney, C. (2002). EEG coherence following acute and chronic clozapine in treatment-resistant schizophrenics. Experimental and Clinical Psychopharmacology, 10(4), 435-444.

Lee, C. W., \& Drummond, P. D. (2008). Effects of eye movement versus therapist instructions on the processing of distressing memories. Journal of Anxiety Disorders, 22, 801-808.

MacCulloch, M. J., \& Feldman, P. (1996). Eye movement treatment utilizes the positive visceral element of the investigatory reflex to inhibit the memories of posttraumatic stress disorder: A theoretical analysis. British Journal of Psychiatry, 169, 571-579.

Maxfield, L., Melnyk, W. T., \& Hayman, C. A. G. (2008). A working memory explanation for the effects of eye movements in EMDR. Journal of EMDR Practice and Research, 2(4), 247-261.

MHyT [logiciel] (2000). Mesa, AZ: Nova Tech EEG. Téléchargé depuis http:/ / www.novatecheeg.com
Mitsar [appareil] (2000). Mesa, AZ: Nova Tech EEG. Téléchargé depuis http: / / www.novatecheeg.com

Nieuwenhuis, S., Elzinga, B. M., Ras, P. H., Berends, F., Duijs, P., Samara, Z., \& Slagter, H. A. (2013). Bilateral saccadic eye movements and tactile stimulation, but not auditory stimulation, enhance memory retrieval. Brain and Cognition, 81, 52-56.

Nunez, P. L., Srinivasan, R., Westdorp, A. F., Wijesinghe, R. S., Tucker, D. M., Silberstein, R. B., \& Cadusch, P. J. (1997). EEG coherency I: Statistics, reference electrode, volume conduction, Laplacians, cortical imaging, and interpretation at multiple sites. Electroencephalography and Clinical Neurophysiology, 103, 499-515.

Oldfield, R. C. (1971). The assessment and analysis of handedness: The Edinburgh Inventory. Neuropsychologia, 9, 97-113.

Pagani, M., Di Lorenzo, G., Verardo, A. R., Nicolais, G., Monaco, L., Lauretti, G., . . Siracusano, A. (2012). Neurobiological correlates of EMDR monitoring-An EEG study. PLoS One, 7(9), 1-12.

Pagani, M., Hogberg, G., Fernandez, I., \& Siracusano, A. (2013). Correlates of EMDR therapy in functional and structural neuroimaging: A critical summary of recent findings. Journal of EMDR Practice and Research, 7(1), 29-38.

Propper, R. E., \& Christman, S. D. (2008). Interhemispheric interaction and saccadic horizontal eye movements: Implications for episodic memory, EMDR, and PTSD. Journal of EMDR Practice and Research, 2, 269-281.

Propper, R. E., Pierce, J., Geisler, M. W., Christman, S. D., \& Bellorado, N. (2007). Effect of bilateral eye movements on frontal interhemispheric gamma EEG coherence: Implications for EMDR therapy. The Journal of Nervous and Mental Disease, 195(9), 785-788.

Rasolkhani-Kalhorn, T., \& Harper, M. (2006). EMDR and low frequency stimulation of the brain. Traumatology, 12(1), 9-24.

Samara, Z., Elzinga, B. M., Slagter, H. A., \& Nieuwenhuis, S. (2011). Do horizontal saccadic eye movements increase interhemispheric coherence? Investigation of a hypothesized neural mechanism underlying EMDR. Frontiers in Psychiatry, 2(4), 1-9.

Servan-Schreiber, D. (2000). Eye movement desensitization and reprocessing: Is psychiatry missing the point? Psychiatric Times, 17, 36-40.

Shapiro, F. (1989). Efficacy of the eye movement desensitization procedure in the treatment of traumatic memories. Journal of Traumatic Stress Studies, 2, 199-223.

Solis-Ortiz, S., Ramos, J., Arce, C., Guevara, M. A., \& CorsiCabrera, M. (1994). EEG oscillations during menstrual cycle. International Journal of Neuroscience, 76, 279-292.

Stevens, L. C., Brady, B., Goon, A., Adams, D., Rebarchik, J., Gacula, L., . . Verdugo, S. (2004). Electrophysiological alterations during hypnosis for ego-enhancement: A preliminary investigation. American Journal of Clinical Hypnosis, 46(4), 323-344.

Stickgold, R. (2002). EMDR: A putative neurobiological mechanism of action. Journal of Clinical Psychology, 58(1), 61-75. 
Tabachnick, B. G., \& Fidell, L. S. (2013). Using multivariate statistics (6th ed.). Upper Saddle River, NJ: Pearson.

Tanev, K. S., Pentel, K. Z., Kredlow, M. A., \& Charney, M. E. (2014). PTSD and TBI co-morbidity: Scope, clinical presentation and treatment options. Brain Injury, 28(3), 261-270.

Van den Hout, M. A., Eidhof, M. B., Verboom, J., Littel, M., \& Engelhard, I. M. (2013). Blurring of emotional and non-emotional memories by taxing working memory during recall. Cognition \& Emotion, 28, 717-727.

Van den Hout, M. A., \& Engelhard, I. M. (2012). How does EMDR work? Journal of Experimental Psychopathology, 3(5), 724-738.

Van den Hout, M. A., Muris, P., Salemink, E., \& Kindt, M. (2001). Autobiographical memories become less vivid and emotional after eye movements. British Journal of Clinical Psychology, 40, 121-130.

Vasterling, J. J., Verfaellie, M., \& Sullivan, K. D. (2009). Mild traumatic brain injury and posttraumatic stress disorder in returning veterans: Perspectives from cognitive neuroscience. Clinical Psychology Review, 29, 674-684.

Weiss, S., \& Mueller, H. M. (2003). The contribution of EEG coherence to the investigation of language. Brain and Language, 85, 325-343.
WinEEG [logiciel] (2000). Mesa, AZ: Nova Tech EEG. Téléchargé depuis http:/ / www.novatecheeg.com

Remerciements. Cette étude a été menée dans le cadre d'une thèse de Master en sciences (M.Sc.) en psychologie de la santé clinique à l'université de l'Arizona du Nord par le premier auteur (MY) sous la supervision du deuxième auteur (LS). Certaines parties de cette étude ont été présentées par MY lors du $91^{\mathrm{e}}$ congrès de la Western Psychological Association qui s'est tenu les 28 avril-1 mai 2011 à Los Angeles, en Californie, et lors du $19^{\mathrm{e}}$ congrès annuel de l'International Society for Neurofeedback and Research le 15 septembre 2011 à Phoenix, en Arizona, ainsi que par le neuvième auteur (BK) lors du $119^{\mathrm{e}}$ congrès annuel de l'American Psychological Association, le 3 août 2001 à Washington, DC.

Nous remercions Madame Noel Larson et Dr Sarah Wyckoff de SenseLabs pour leur aide dans la génération de cartes de cohérence pour cette étude.

Merci d'adresser tout correspondance concernant cet article à Larry Stevens, $\mathrm{PhD}$, Department of Psychological Sciences, NAUBox 15106, NorthernArizona University, Flagstaff, AZ 86011,États-Unis.Courriel:Larry.Stevens@nau.edu 